\title{
C. ELEGANS PEZO-1 IS A Mechanosensitive ChANNEL INVOLVED IN FoOd SENSATION
}

6 Jonathan RM Millet ${ }^{1,2}$, Luis O Romero ${ }^{1,3}$, Jungsoo Lee ${ }^{1}$, and Valeria Vásquez ${ }^{1, *}$.

$9{ }^{1}$ Department of Physiology, College of Medicine, University of Tennessee Health Science Center,

10 Memphis, TN 38163, USA

$11{ }^{2}$ Present address: Institute of Neuroscience, Department of Biology, University of Oregon, Eugene,

12 OR 97403-1254, USA

$13{ }^{3}$ Integrated Biomedical Sciences Graduate Program, College of Graduate Health Sciences,

14 University of Tennessee Health Science Center, Memphis, TN 38163, USA 


\section{ABSTRACT}

PIEZO channels are force sensors essential for physiological processes including baroreception

27 and proprioception. The Caenorhabditis elegans genome encodes an ortholog gene of the Piezo

28 family, pezo-1, expressed in several tissues including the pharynx. This myogenic pump is an essential

29 component of the $C$. elegans alimentary canal whose contraction and relaxation are modulated by

30 mechanical stimulation elicited by food content. Whether pezo- 1 encodes a mechanosensitive channel

31 and contributes to pharyngeal function remains unknown. Here, we leverage genome editing, genetics,

32 microfluidics, and electropharyngeogram recordings to establish that pezo- 1 is expressed in the

33 pharynx, including a proprioceptive-like neuron, and regulates pharyngeal function. Knockout (KO)

34 and gain-of-function (GOF) mutants reveal that pezo- 1 is involved in fine-tuning pharyngeal pumping

35 frequency, sensing osmolarity and food quality. Using pressure-clamp experiments in primary $C$.

36 elegans embryo cultures, we determine that pezo- $1 \mathrm{KO}$ cells do not display mechanosensitive currents,

37 whereas cells expressing wild-type or GOF PEZO-1 exhibit mechanosensitivity. Moreover, infecting

38 the Spodoptera frugiperda cell line with a baculovirus containing the pezo- 1 isoform G (among the

39 longest isoforms) demonstrates that pezo-1 encodes a mechanosensitive channel. Our findings reveal

40 that pezo- 1 is a mechanosensitive ion channel that regulates food sensation in worms. 
Mechanosensitive ion channels regulate several physiological processes ranging from osmotic balance in bacteria (Kung, Martinac and Sukharev, 2010), turgor control in plants (Hamilton, Schlegel and Haswell, 2015), touch (Geffeney and Goodman, 2012; Yan et al., 2013; Ikeda et al., 2014; Maksimovic et al., 2014; Ranade et al., 2014; Woo et al., 2014; Chesler et al., 2016), pain (Murthy et al., 2018; Szczot et al., 2018), proprioception (Woo et al., 2015), hearing (Pan et al., 2018), lineage choice (Pathak et al., 2014), and blood pressure regulation in animals (Retailleau et al., 2015; Wang et al., 2016; Rode et al., 2017; Zeng et al., 2018). These channels are ubiquitous, as they transduce mechanical stimuli into electrochemical signals in all kingdoms of life (Kung, Martinac and Sukharev, 2010; Geffeney and Goodman, 2012; Douguet and Honoré, 2019). In 2010, the PIEZO1 and PIEZO2 channels were identified as essential components of distinct, mechanically activated cation channels in mammalian cells (Coste et al., 2010). Since then, many physiological roles have been assigned to these two ion channels (Parpaite and Coste, 2017).

Mammalian PIEZO channels have been associated with several hereditary pathophysiologies (Alper, 2017). Piezo1 gain-of-function (GOF) mutations display slow channel inactivation leading to an increase in cation permeability and subsequent red blood cell dehydration (Zarychanski et al., 2012; Albuisson et al., 2013; Bae et al., 2013; Ma et al., 2018). For instance, the human Piezo1 hereditary mutation $\mathrm{R} 2456 \mathrm{H}$ located in the pore domain decreases inactivation and, when substituted by Lys inactivation is completely removed (Bae et al., 2013). Piezo1 global knockouts (KO) are embryonically lethal in mice (Li et al., 2014; Ranade et al., 2014) and cell-specific KOs result in animals with severe defects (Wu, Lewis and Grandl, 2017; Ma et al., 2018). Intriguingly, both Piezo2 $\mathrm{KO}$ and GOF mutations are associated with joint contractures, skeletal abnormalities and alterations in muscle tone (Coste et al., 2013; Chesler et al., 2016; Yamaguchi et al., 2019). GOF and loss-offunction (LOF) mutations are useful genetic tools to determine the contribution of PIEZO channels to mechanosensation in various physiological processes and in different animals. 
The C. elegans genome encodes an ortholog of the Piezo channel family, namely pezo-1

(wormbase.org v. WS280). Recently, Bai and collaborators showed that pezo- 1 is expressed in several

tissues including the pharynx (Bai et al., 2020). The worm's pharynx is a pumping organ that

rhythmically couples muscle contraction and relaxation in a swallowing motion to pass food down to

73 the animal's intestine (Keane and Avery, 2003). This swallowing motion stems from a constant low-

74 frequency pumping maintained by pharyngeal muscles and bursts of high-frequency pumping from a dedicated pharyngeal nervous system (Avery and Horvitz, 1989; Raizen, Lee and Avery, 1995; Trojanowski, Raizen and Fang-Yen, 2016; Lee et al., 2017). In mammals, the swallowing reflex is initiated when pressure receptors in the pharynx walls are stimulated by food or liquids, yet the identity of the receptor(s) that directly evoke this mechanical response remain to be identified (Tsujimura et al., 2019). Interestingly, the Drosophila melanogaster PIEZO ortholog is a mechanosensitive ion channel (Kim et al., 2012) required for feeding while also avoiding food over-consumption (Min et al., 2020; Wang et al., 2020). To date, whether pezo-1 encodes for a mechanosensitive ion channel or regulates worm's pharyngeal activity has yet to be determined.

Here, we found a strong and diverse expression of the pezo-1 gene in pharyngeal tissues by imaging a pezo-1::GFP transgenic reporter strain. By leveraging genetic dissection, electrophysiological measurements, and behavior analyses, we also established that PEZO-1 is required for proper low frequency electric activity and pumping behavior. Analyses of pezo-1 KO and GOF mutants demonstrated that decreasing or increasing PEZO-1 function upregulates pharyngeal pumping frequency. Likewise, mutants display distinct pharyngeal activities triggered by the neurotransmitter serotonin or with various buffer osmolarities. By using elongated bacteria as a food source, we demonstrated that pezo- $1 \mathrm{KO}$ decreases pharyngeal pumping frequency, whereas the GOF mutant increases it. Finally, electrophysiological recordings of pezo-1 expressing cells from C. elegans embryo cultures and Spodoptera frugiperda (Sf9) cell line demonstrate that pezo-1 encodes a mechanosensitive ion channel. Altogether, our results show that pezo-1 is a mechanosensitive ion 


\section{Strains and Maintenance}

Worms were propagated as previously described (Brenner, 1974). N2 (var. Bristol) was

referred as wild type (WT) throughout the manuscript. The following strains were used: VVR3 unc119(ed3)III;decEx1(pRedFlpHgr)(C10C5.1[20789]::S0001_pR6K_Amp_2xTY1ce_EGFP_FRT_ rpsl_neo_FRT_3xFlag)dFRT::unc-119-Nat, COP1553 (KO: 6,616 bp deletion) pezo-1 (knu508) IV, COP1524 (GOF: R2373K) pezo-1 (knu490) IV, LX960 lin-15B\&lin-15A(n765) X; vsIs97 [tph1p::DsRed2 + lin-15(+)], DA572 eat-4(ad572) III, and DA1051 avr-15(ad1051) V. Transgenic strain VVR3 was obtained by microinjecting a fosmid construct (from the TransgeneOme Project) in a unc119(ed3) strain from InVivo Biosystems. COP1553 and COP1524 were obtained using the CRISPR-

Cas9 method (InVivo Biosystems). Transgenic worm VVR3 expressing GFP under the control of Ppezo-1::GFP was crossed with pezo-1 mutants COP1553 and COP1524 to obtain VVR69 and

VVR70, respectively. LX960 was kindly provided by Dr. Kevin Collins (University of Miami).

\section{Imaging}

Worms were selected individually and dropped in $15 \mu \mathrm{L}$ of $\mathrm{M} 9$ buffer $(86 \mathrm{mM} \mathrm{NaCl}, 42 \mathrm{mM}$

$110 \mathrm{Na}_{2} \mathrm{HPO}_{4}, 22 \mathrm{mM} \mathrm{KH}_{2} \mathrm{PO}_{4}, 1 \mathrm{mM} \mathrm{MgSO}$ ) paralyzed on a glass slide containing $2 \%$ agarose pads 111 containing $150 \mathrm{mM}$ 2,3-butanedione monoxime (BDM). Bright field and fluorescence imaging were 112 done on a Zeiss 710 Confocal microscope using either a 20X or 40X objective. Images were processed 113 using Fiji ImageJ (Schindelin et al., 2009) to enhance contrast and convert to an appropriate format.

\section{Worms' synchronization}

For all pharyngeal pumping assays, worms were synchronized by picking young adults onto

116 fresh nematode growth media (NGM) plates seeded with E coli strain OP50 and left to lay eggs for 117 two hours at $20^{\circ} \mathrm{C}$. Then, the adults were removed, and the plates incubated at $20^{\circ} \mathrm{C}$ for three days. 


\section{Pharyngeal pumping}

Serotonin profile. A serotonin aliquot (InVivo Biosystems) was diluted in M9 Buffer prior to experiments and discarded after three hours. 42 synchronized worms were picked and transferred in $200 \mu \mathrm{L}$ of M9 Buffer supplemented with 2-, 5-, 10- or $20-\mathrm{mM}$ serotonin and incubated at $20^{\circ} \mathrm{C}$ for 30 minutes before being loaded inside the microfluidic chip (SC40, The ScreenChip ${ }^{\text {TM }}$ System, InVivo Biosystems).

Control E. coli assay. OP50 was grown in liquid LB medium under sterile conditions at $37^{\circ} \mathrm{C}$ and diluted to an optical density of 1.0 . Bacterial cultures were stored at $4{ }^{\circ} \mathrm{C}$ for up to a week.

Spaghetti-like E. coli assay. OP50 colonies were picked from a fresh LB plate and incubated in $2 \mathrm{~mL}$ of LB overnight the day before the experiment. The following day, $0.5 \mathrm{~mL}$ of the preincubation culture was used to inoculate $1.5 \mathrm{~mL}$ of LB media and grown until growth was exponential, which was verified by checking optical density (optical density of 0.5 ). Cephalexin (Alfa Aesar ${ }^{\mathrm{TM}}$ ) was then added at $60 \mu \mathrm{g} / \mathrm{ml}$ final concentration and the culture was incubated for two hours. Spaghettilike OP50 were verified under a microscope and washed three times using $2 \mathrm{~mL}$ of M9 buffer followed by centrifugation at $400 \mathrm{~g}$ to gently pelletize the elongated bacteria.

Pharyngeal recordings and Analyses. Worms were loaded one-by-one inside the microfluidic chip recording channel and left to adjust for one minute prior to recording. All recordings were two minutes long. Records were analysed using NemAnalysis software (InVivo Biosystems) with the brute force algorithm turned off. Parameters were adjusted for each record in order to include the maximum numbers of clearly identifiable pharyngeal pumps. Results were exported from the software in sheet form and parameters were plotted and statistically analysed using MATLAB R2019a (MathWorks).

\section{Development assay} Young adults were allowed to lay eggs on NGM plates seeded with control or spaghetti-like bacteria for two hours. Spaghetti-like bacteria were cultured as described above. Animals (10-20 
142 worms) were removed for plates after two hours and the number of eggs laid was counted. After 3

143 days of incubation, animals that reached adulthood were counted in each trial, and results were 144 compared across four trials.

\section{Food ingestion assay}

A drop of fresh cultured of control or spaghetti-like bacteria with $2 \mu \mathrm{M}$ DiI dye (Sigma, CAS \#41085-99-8) was placed on a NGM agar plate. Young adults were fed bacteria with DiI for 30 min.

148 Worms were transferred into OP50 seeded NGM without dye for 5 min (Vidal-Gadea et al., 2012).

149 Animals were placed in a thin-layered BDM-agarose plate for imaging under a Nikon SMZ18 stereomicroscope. Food occupation in the digestive tract was detected by fluorescence.

\section{Primary culture of $C$. elegans embryo cells}

C. elegans embryonic cells were generated as previously described (Strange, Christensen and

Morrison, 2007). Worms were grown on $10 \mathrm{~cm}$ enriched peptone plate with NA22 E. coli. NA22

bacteria grow in very thick layers that provide abundant food source for large quantities of worms.

The synchronized gravid hermaphrodites were bleached to release eggs and washed with sterile egg buffer (118 mM NaCl, $48 \mathrm{mM} \mathrm{KCl,} 2 \mathrm{mM} \mathrm{CaCl}_{2}, 2 \mathrm{mM} \mathrm{MgCl} 2,25 \mathrm{mM}$ HEPES, pH 7.3, 340 mOsm, adjusted with sucrose). The isolated eggs were separated from debris by centrifugation in a $30 \%$ sucrose solution. Chitinase ( $1 \mathrm{U} / \mathrm{ml}$, Sigma) digestion was performed to remove eggshells. The embryo cells were dissociated by pipetting and filtered through a sterile $5 \mu \mathrm{m}$ Durapore filter (Millipore). The cells were plated on glass coverslips coated with peanut lectin solution (SIGMA; $0.5 \mathrm{mg} / \mathrm{ml}$ ) and cultured in L15 media (Gibco) supplemented with $50 \mathrm{U} / \mathrm{ml}$ penicillin, $50 \mu \mathrm{g} / \mathrm{ml}$ streptomycin with $10 \%$ fetal bovine serum (FBS, Invitrogen) for $72-96 \mathrm{hrs}$.

\section{Expression of pezo-1 in Sf9 insect cells}

We generated a baculovirus construct consisting of an $8 \times$ histidines-maltose binding protein 
166

167

168

169

according to RNA sequencing, wormbase.org v. WS280). We infected Sf9 cells with the pezo-1 containing baculovirus for 48 hours. Infected cells were plated on glass coverslips coated with a peanut lectin solution (SIGMA; $0.5 \mathrm{mg} / \mathrm{ml}$ ) for patch-clamp experiments.

\section{Electrophysiology and mechanical stimulation}

Primary cultured embryo cells labeled with Ppezo-1::GFP from strains VVR3, VVR69, or VVR70 were recorded in the cell-attached configuration of the patch clamp technique. Control and infected Sf9 insect cells were recorded in the whole-cell patch clamp configuration. For on-cell recordings, the bath solution contained $140 \mathrm{mM} \mathrm{KCl}, 6 \mathrm{mM} \mathrm{NaCl}, 2 \mathrm{mM} \mathrm{CaCl}_{2}, 1 \mathrm{mM} \mathrm{MgCl}_{2}, 10 \mathrm{mM}$ glucose, and $10 \mathrm{mM}$ HEPES (pH 7.4; 340 mOsm, adjusted with sucrose). The pipette solution contained $140 \mathrm{mM} \mathrm{NaCl}, 6 \mathrm{mM} \mathrm{KCl}, 2 \mathrm{mM} \mathrm{CaCl}_{2}, 1 \mathrm{mM} \mathrm{MgCl} 2,10 \mathrm{mM}$ glucose, and $10 \mathrm{mM}$ HEPES (pH 7.3; 330 mOsm, adjusted with sucrose). Pipettes were made out of borosilicate glass (Sutter Instruments) and were fire-polished before use until a resistance between 3 and $4 \mathrm{M} \Omega$ was reached. Currents were recorded at a constant voltage $(-60 \mathrm{mV}$, unless otherwise noticed), sampled at $20 \mathrm{kHz}$, and low pass filtered at $2 \mathrm{kHz}$ using a MultiClamp 700B amplifier and Clampex (Molecular Devices, LLC). Leak currents before mechanical stimulations were subtracted offline from the current traces. Cells were mechanically stimulated with negative pressure applied through the patch pipette using a High-Speed Pressure Clamp (ALA Scientific) automated using a MultiClamp 700B amplifier through Clampex (Molecular Devices, LLC). Cell-attached patches were probed using a square-pulse protocol consisting of $-10 \mathrm{mmHg}$ incremental pressure steps, each lasting $1 \mathrm{~s}$ in $10 \mathrm{~s}$ intervals. Cells which gigaseals did not withstand at least six consecutive steps of mechanical stimulation were excluded from analyses. Isteady was defined as the maximal current in the steady state. Deactivation was compared by determining the percentage of $\mathrm{I}_{\text {steady }}$ left $100 \mathrm{~ms}$ after the mechanical stimuli ended.

For whole-cell recordings, the bath solution contained $140 \mathrm{mM} \mathrm{NaCl}, 6 \mathrm{mM} \mathrm{KCl}, 2 \mathrm{mM} \mathrm{CaCl}_{2}$, $1 \mathrm{mM} \mathrm{MgCl} 2,10 \mathrm{mM}$ glucose, and $10 \mathrm{mM}$ HEPES (pH 7.4). The pipette solution contained $140 \mathrm{mM}$ $\mathrm{CsCl}, 5 \mathrm{mM} \mathrm{EGTA}, 1 \mathrm{mM} \mathrm{CaCl}_{2}, 1 \mathrm{mM} \mathrm{MgCl}$, and $10 \mathrm{mM}$ HEPES (pH 7.2). For indentation assays, 
191 Sf9 cells were mechanically stimulated with a heat-polished blunt glass pipette $(3-4 \mu \mathrm{m})$ driven by a

192 piezo servo controller (E625, Physik Instrumente). The blunt pipette was mounted on a 193 micromanipulator at an $\sim 45^{\circ}$ angle and positioned $3-4 \mu \mathrm{m}$ above from the cells without indenting

194 them. Displacement measurements were obtained with a square-pulse protocol consisting of $1 \mu \mathrm{m}$ 195 incremental indentation steps, each lasting $200 \mathrm{~ms}$ with a $2 \mathrm{~ms}$ ramp in $10 \mathrm{~s}$ intervals. Recordings 196 with leak currents $>200 \mathrm{pA}$, with access resistance $>10 \mathrm{M} \Omega$, and cells which giga-seals did not 197 withstand at least five consecutive steps of mechanical stimulation were excluded from analyses.

Data and fits were plotted using OriginPro (from OriginLab). Sigmoidal fit was done with the 199 following Boltzmann equation:

Eq. 1: $f_{(x)}=A_{2}+\frac{A_{1}-A_{2}}{\left.1+e^{\left(X-X_{O}\right)} / d X\right)}$

201 where $\mathrm{A}_{2}=$ final value, $\mathrm{A}_{1}=$ initial value; $\mathrm{X}_{\mathrm{o}}=$ center, and $d X=$ time constant.

\section{Data and Statistical analyses}

Data and statistical analyses were performed using DataGraph 4.6.1, MATLAB R2019a

204 (MathWorks), and GraphPad Instat 3 software. Statistical methods and sample numbers are detailed 205 in the corresponding figure legends. No technical replicates were included in the analyses. 


\section{$\underline{\text { RESULTS }}$}

pezo-1 is expressed in a wide variety of cells in the worm's pharynx

To determine the expression of pezo-1 in C. elegans, we used a fluorescent translational

210 reporter made by the TransgeneOme Project (Hasse, Hyman and Sarov, 2016). This fosmid construct

211 contains pezo-1 native cis-regulatory elements, including introns, up to exon 17 and 3' UTR sequences

212 linked in-frame to the green fluorescent protein (GFP; Figure 1A). The position of the GFP with

213 respect to the remainder of the gene creates an unnatural truncated version of the PEZO-1 protein.

214 Hence, it likely expresses a non-functional protein that misses 16 exons containing the majority of the

215 pezo- 1 sequence (including the pore domain). GFP signals are present in all developmental stages and

216 multiple cells (Supplementary Figure 1A-B), and it does not appear to be mosaic as similar expression

217 patterns were observed in at least three independent transgenic lines. We imaged pezo-1::GFP worms

218 at different focal planes to identify the different cells expressing GFP based on their morphological

219 features (i.e., cell-bodies position, neurites extension and positions along the body, and branching).

220 The strongest GFP signals that we identified come from the pharyngeal gland cells (Figure 1B, bright

221 and fluorescence fields). These cells are composed of five cell bodies (two ventral g1s, one dorsal g1

222 and two ventral g2s) located inside the pharynx terminal bulb and three anterior cytoplasmic

223 projections: two shorts that are superposed ending in the metacorpus and a long one reaching the end

224 of the pm3 muscle. These cells are proposed to be involved in digestion (Albertson and Thomson,

225 1976; Ohmachi et al., 1999), lubrication of the pharynx (Smit, Schnabel and Gaudet, 2008), generation

226 and molting of the cuticle (Singh and Sulston, 1978; Höflich et al., 2004), and resistance to pathogenic

227 bacteria (Höflich et al., 2004). Additionally, we visualized, pezo-1::GFP in a series of cells

228 surrounding the muscle of the corpus and the isthmus (Figure 1C) whose morphology and location

229 match with those of the arcade cells; these were previously hypothesized to be support cells (Albertson

230 and Thomson, 1976). We also recognized as putative pezo-1 expressing cells: glial cell AmSh,

231 pharyngeal interneuron $\mathrm{I} 3$ (Figure 1D), and $\mathrm{M} 3 \mathrm{~L}_{\mathrm{L} / \mathrm{R}}$ somas and neurites (Figure 1E-F). These neurons 
232 are involved in pharyngeal pumping relaxation (Avery, 1993). The finger-like structure known as the

233 pharyngeal sieve, at the junction between corpus and isthmus, also expresses pezo-1 (Figure 1F).

By crossing pezo- $1:: G F P$ with a $t p h-1::$ DsRed marker carrying strain, we were able to identify

235 pezo- 1 expression in the pharyngeal $\mathrm{NSM}_{\mathrm{L} / \mathrm{R}}$ secretory, motor, and sensory neurons (Figure $1 \mathrm{G}$ ).

236 Importantly, these serotoninergic neurons have been proposed to sense food in the lumen of the

237 pharynx through their proprioceptive-like endings and trigger feeding-related behaviors (i.e., increased

238 pharyngeal pumping, decreased locomotion, and increased egg laying) (Albertson and Thomson,

239 1976; Avery, Bargmann and Horvitz, 1993). In addition to the pharyngeal cells, we observed 240 expression of pezo-1 in the ventral nerve cord (VNC, Figure 1D) neurons, striated muscles

241 (Supplementary Figure 1C), coelomocytes (Supplementary Figure 1D-E), spermatheca

242 (Supplementary Figure 1B and 1E), vulval muscles (Supplementary Figure 1F), and various male

243 neurons including the ray neurons (Supplementary Figure 1G). Importantly, the expression pattern

244 reported by our pezo- 1 fosmid construct matches very well with the gene expression atlas for $C$.

245 elegans neurons (Taylor et al., 2020), except for the M3 neurons. The strong and varied pezo-1

246 expression in the pharynx along with the function of the cells expressing it, led us to investigate the

247 potential contribution of PEZO-1 to pharyngeal function. 
A
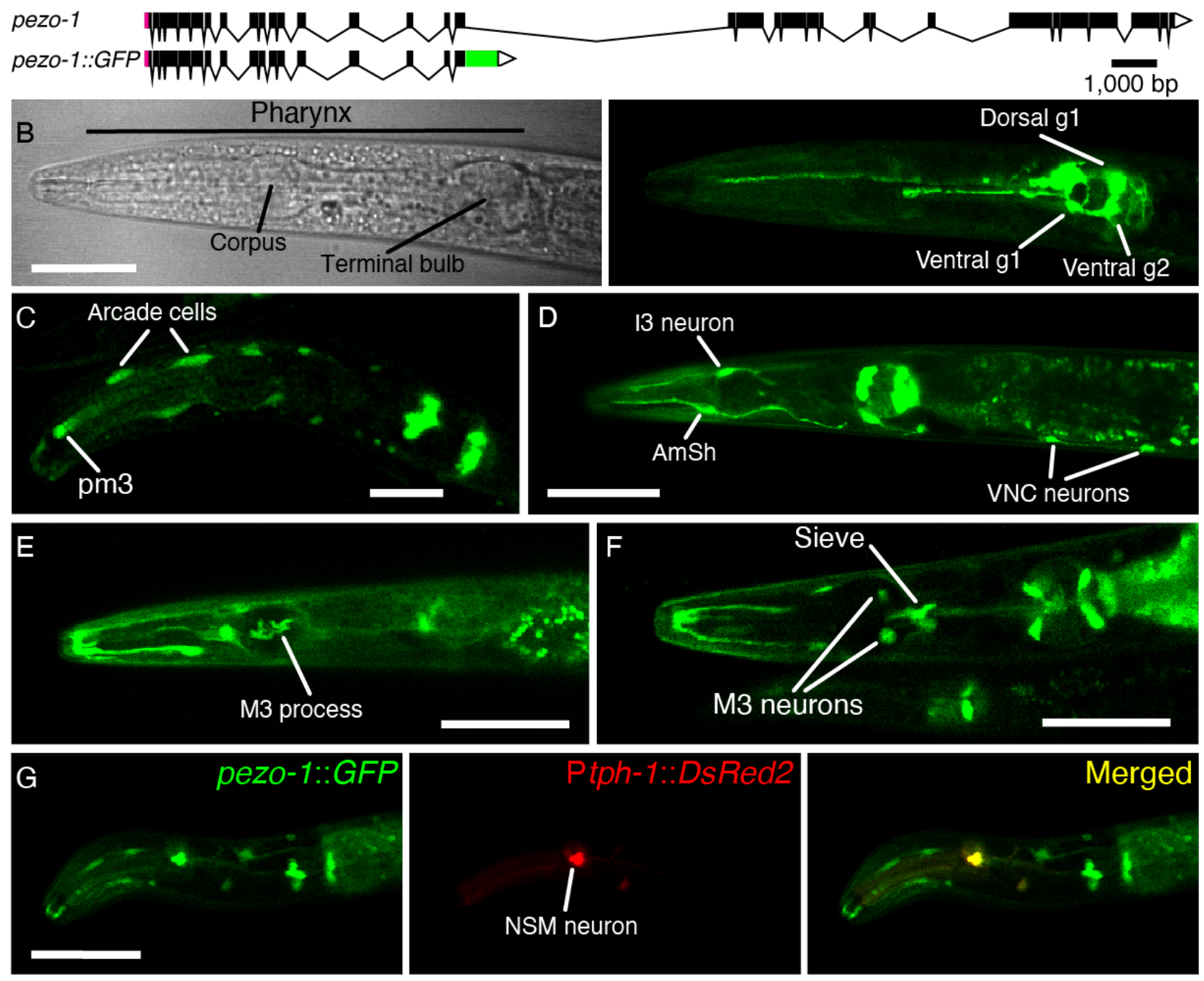

Figure 1. pezo-1 is strongly expressed in C. elegans pharynx. (A) pezo-1 gene diagram according to wormbase.org, v. WS280 made with Exon-Intron Graphic Maker http://wormweb.org/. Magenta rectangles and white triangles denote the 5' and 3' untranslated regions (UTR), respectively. Black rectangles denote exons and black lines denote introns. Green rectangle denotes the GFP sequence inserted after exon 17. (B) Brightfield (left) and fluorescence (right) micrographs of the anterior end of a young adult pezo-1::GFP hermaphrodite highlighting pharynx structures and the GFP reporter expression in gland cells. Scale bar represents $50 \mu \mathrm{m}$. (C) Micrograph of the anterior end of a young adult pezo-1::GFP hermaphrodite expressing GFP in cells identified as arcade and pharyngeal muscle cells (pm3), according to their position. Scale bar represents $20 \mu \mathrm{m}$. (D-E) Micrograph of the anterior end of a young adult pezo- $1::$ GFP hermaphrodite expressing GFP in cells identified as neurons (I3, M3, VNCs) and sheath cell (AmSh), according to their position. Scale bar represents $50 \mu \mathrm{m}$. (F) Micrograph of the anterior end of a young adult pezo-1::GFP hermaphrodite expressing GFP in cells identified ad M3 and pharyngeal sieve, according to their position. Scale bar represents $100 \mu \mathrm{m}$. (G) Co-localization between $t p h-1::$ DsRed2 and pezo-1::GFP reporter in the neuron NSM. Scale bar represents $50 \mu \mathrm{m}$. Micrographs are representative of at least 20 independent preparations. 


\section{Serotonin stimulation reveals different pharyngeal pump parameters}

To analyze the contribution of pezo- 1 to pharyngeal pumping in $C$. elegans, we used the ScreenChip ${ }^{\mathrm{TM}}$ system (InVivo Biosystems) that allows measuring electropharyngeogram recordings

254 (EPG; Figure 2A) (Raizen and Avery, 1994) by loading single-live worms inside a microfluidic chip.

Figure 2A-B summarizes the pharynx anatomy, electrical properties measured during an EPG, and the neurons involved in pharyngeal function. For instance, the excitation event (E spike) precedes the pharyngeal contraction and is modulated by the pacemaker neuron $\mathrm{MC}$ (Figure $2 \mathrm{~B}$, top), whereas the repolarization event ( $\mathrm{R}$ spike) leads to pharyngeal relaxation and correlates to the activity of the inhibitory M3 motorneurons (Figure 2B, middle). Every 3-4 pumps, there is relaxation of the terminal bulb (isthmus peristalsis) which is modulated by the motorneuron M4 (Figure 2B, bottom) (Avery and Horvitz, 1989). The main EPG events are regulated by the pharyngeal proprioceptive neuron NSM. Importantly, M3 motorneurons (potentially) and the NSM proprioceptive neuron express pezo-1 (Figure 1E-G).

Analyses of the EPG records allow determination of different pharyngeal pumping parameters including frequency, duration, and the time interval that separates two pumping events (hereafter referred to as the interpump interval). We used serotonin to increase pharyngeal activity since, in the absence of food or serotonin, the pumping events are infrequent. Serotonin mimics food stimulation by activating the $M C_{L / R}$ and $M 3_{L / R}$ neurons (Niacaris, 2003). First, we established a serotonin doseresponse profile of the WT strain pharyngeal pumping parameters (N2; Figure 2C-G). Serotonin increases pharyngeal pumping frequency in a dose-dependent manner, with concentrations above 5 $\mathrm{mM}$ increasing the likelihood of reaching $5 \mathrm{~Hz}$ (Figure 2C). We averaged the EPG recordings at each serotonin concentration and found a clear difference in pump durations between 0- and 5-mM.

273 Concentrations equal or higher than $5 \mathrm{mM}$ evoke similar pump durations ( 100 ms; Figure 2D).

274 Interestingly, analyses of the pump duration distribution profile under serotonin stimulation revealed 
A

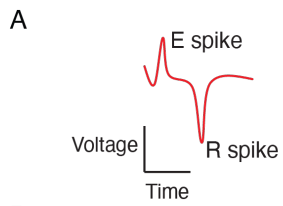

B
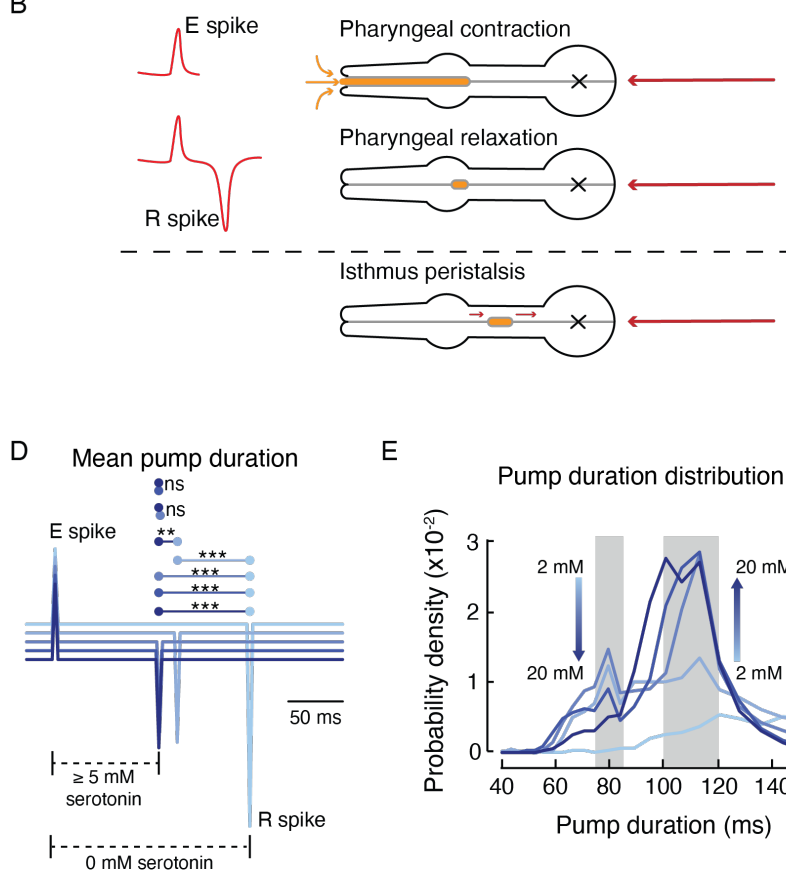
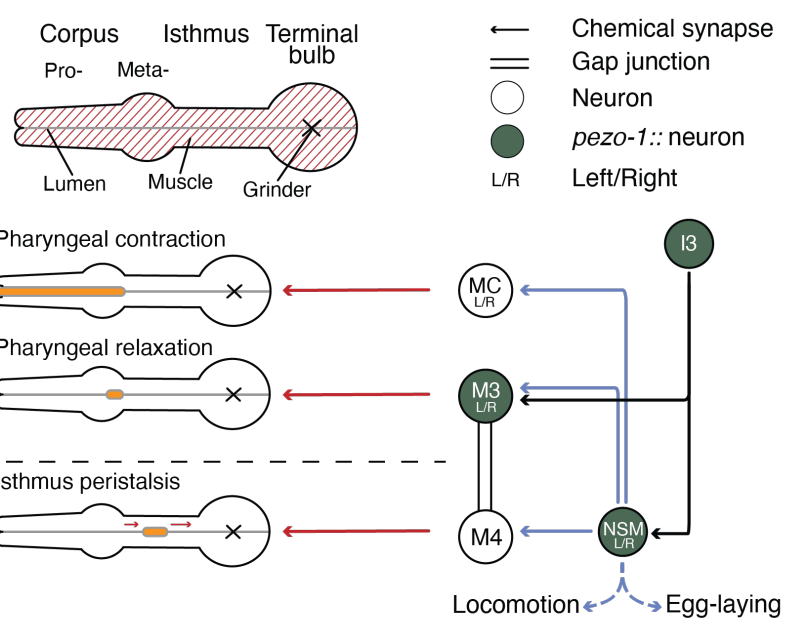

E

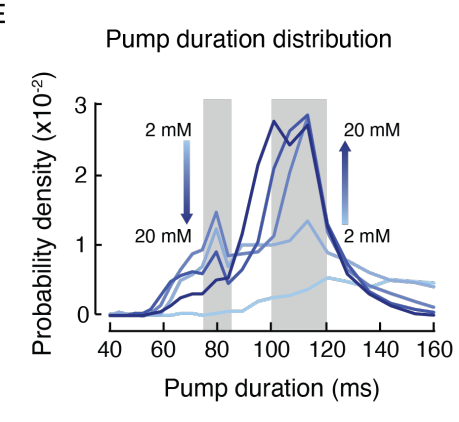

$\mathrm{F}$

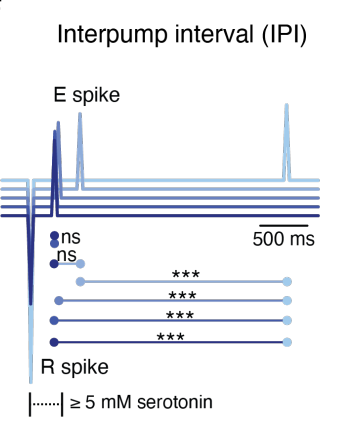

C
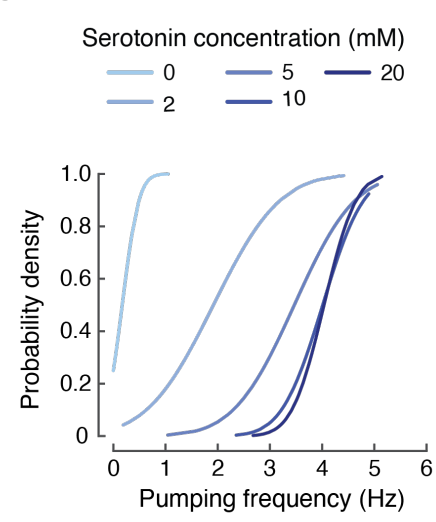

G

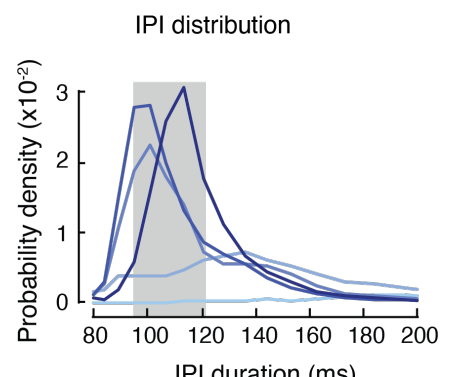

Figure 2. Serotonin triggers pharyngeal pumping in a concentration-dependent manner in WT (N2) worms. (A) Left, illustration of an electropharyngeogram (EPG) recording depicting the contraction-relaxation of the corpus, referred to as a pharyngeal pump. Right, illustration representing C. elegans pharyngeal muscle. (B) Representation of the main pharyngeal electrical coupling in $C$. elegans. Blue arrows represent serotoninergic transmission, red arrows represent neuromuscular junction, double lines represent gap junction, filled circles represent neurons putatively expressing of pezo-1, open circles represent neurons not expressing pezo-1. (C) Probability densities for pharyngeal pumping frequencies at different serotonin concentrations. Results for every serotonin concentration were statistically compared by Kolmogorov-Smirnov test ( $p$-values are shown in Table I). $\mathrm{n}$ is 90 worms per condition. (D) Ensemble averages of EPG traces for different serotonin concentrations showing pump durations (E to R spikes). One-way ANOVA and Tukey-Kramer multiple comparisons test. $\mathrm{n}$ is 90 worms per condition (E) Serotonin concentration effect on pump duration. A kernel probability distribution was fit to the data. $\mathrm{n}$ is 90 worms per condition. (F) Ensemble averages of EPG traces for different serotonin concentrations showing interpump interval ( $R$ to $E$ spikes). One-way ANOVA and Tukey-Kramer multiple comparisons test. $\mathrm{n}$ is 90 worms per condition (G) Serotonin concentration effect interpump interval. A kernel probability distribution was fit to the data. $\mathrm{n}$ is 90 worms per condition. Asterisks indicate values significantly different $(* * * p<0.001$ and $* * p<0.01)$ and ns indicates not significantly different.

278 We observed that the fast and slow categories displayed an inverse relationship with respect to serotonin concentration (Figure 2E; arrows). We hypothesize that slow (100-120 ms) pumps are the result of the serotoninergic activation of $\mathrm{M} 3_{\mathrm{L} / \mathrm{R}}$ while the fast $(\sim 80 \mathrm{~ms})$ pumps originate from a 
mechanism that is partly independent from the serotonin pathway. Unlike pump duration, we observed only one category for interpump intervals around 95-120 ms for 5- to 20-mM serotonin concentrations (Figure 2F-G). Interestingly, we did not observe inter pump intervals faster than $90 \mathrm{~ms}$, regardless of the serotonin concentration. The inter pump intervals results support the idea that there is a minimum refractory period between two pumps. This set of analyses allowed us to establish a suitable model for evaluating the role of pezo-1 function in vivo.

pezo-1 mutants display higher pumping frequency than WT worms

To determine whether pezo- 1 has a functional role in pharyngeal pumping, we engineered LOF

and GOF mutants. A putative LOF mutant obtained by deleting $6,616 \mathrm{bp}$ from the pezo- 1 locus (hereafter referred to as pezo-1 KO; Figure 3A, top). Previous works demonstrated that the substitution of R2456H (located at the pore helix) of the ortholog human Piezol gene increases cation permeability (GOF) and causes hemolytic anemia (Zarychanski et al., 2012; Albuisson et al., 2013; Bae et al., 2013). Moreover, a conservative substitution of Lys for Arg at position 2456 in the human Piezo1 channel exhibits a pronounced decreased inactivation when compared to the WT or R2456H channels (Bae et al., 2013). Hence, we engineered a putative GOF mutant strain obtained by substituting the conserved Arg 2373 with Lys (hereafter referred to as pezo-1 R2373K or GOF; Figure 3A, bottom). Parenthetically, the R2373K numbering position is based on isoform $\mathrm{G}$ - one of the longest isoforms according to RNA sequencing (wormbase.org v. WS280). We also included in our analysis two mutants known to alter pharyngeal function, eat-4(ad572) and avr-15(ad1051). EAT-4 is a glutamatesodium symporter involved in postsynaptic glutamate reuptake. eat-4(ad572) affects the neurotransmission efficiency of all glutamatergic pharyngeal neurons ( $2_{\mathrm{L} / \mathrm{R}}, \mathrm{I} 5, \mathrm{M} 3_{\mathrm{L} / \mathrm{R}}, \mathrm{M} 4, \mathrm{MI}$, $\mathrm{NSM}_{\mathrm{L} / \mathrm{R}}$ ) (Lee et al., 1999). AVR-15 is a glutamate-gated chloride channel expressed in the pharyngeal muscle pm4 and pm5 (both synapsed by $\mathrm{M} 3_{\mathrm{L} / \mathrm{R}}$ ) and involved in relaxation of the pharynx. Its mutant allele ad1051 lengthen pump duration by delaying relaxation of the pharynx in a similar fashion as 
At $2 \mathrm{mM}$ concentration of exogenous serotonin (to elicit pharyngeal activity), both pezo- $1 \mathrm{KO}$ and R2373K mutants displayed significantly higher pumping frequencies than WT and similar to avr-

309 15(ad1051) (Figure 3B). On the other hand, the eat-4(ad572) mutant displayed lower pumping

310 frequency at this serotonin concentration. To further assess the pezo- 1 mutants' pharyngeal altered

311 function, we analyzed the pump duration distributions from the EPG records. pezo- 1 KO distribution

312 is similar to the WT (Figure 3C, red vs. black) whereas the R2373K mutant profile is reminiscent of

313 the avr-15(ad1051), as both mutant strains displayed a narrower distribution around $100 \mathrm{~ms}$ pump

314 events (Figure 3C, blue and green $v$ s. black). Moreover, the R2373K mutant lacked fast pump events

315 between 50 to $80 \mathrm{~ms}$ (Figure 3C, blue bracket), similar to the WT features observed at high serotonin

316 concentrations ( $\geq 5 \mathrm{mM}$, Figure $2 \mathrm{E}$ ), and the eat-4(ad572) mutant and the avr-15(ad1051) mutant at

$3172 \mathrm{mM}$ serotonin concentration (Figure 3C, yellow and green brackets). The analysis of distribution of 318 interpump intervals revealed that pezo- $1 \mathrm{KO}$ and $\mathrm{R} 2373 \mathrm{~K}$ mutants, although different, both spend less 319 time resting between pumps $(95-120 \mathrm{~ms})$ than the WT $(\approx 140 \mathrm{~ms})$ (Figure $3 \mathrm{D}$, red and blue brackets).

320 This enhancement in function resembles the WT activity measured at 5- to 20-mM serotonin 321 concentrations (Figure $2 \mathrm{~F}-\mathrm{G}$ ) and could account for the increase in frequency shown in Figure 3B. The 322 close resemblance between the PEZO-1 GOF and the avr-15(ad1051) mutants' pharyngeal pumping 323 parameters suggests a potential link between PEZO-1 and pharyngeal relaxation through $\mathrm{M} 3_{\mathrm{L} / \mathrm{R}}$ 324 neurotransmission. 
A

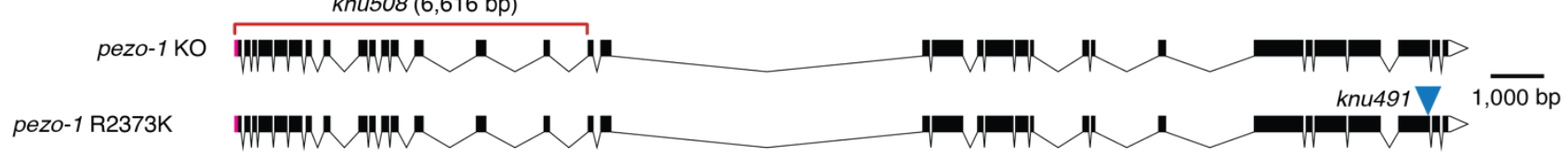

B

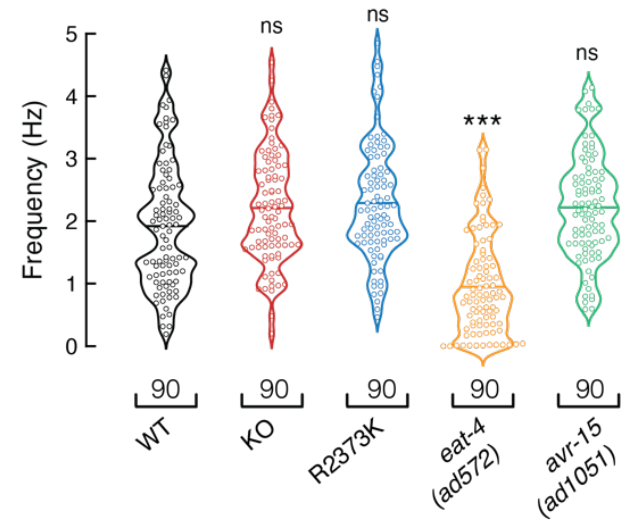

D

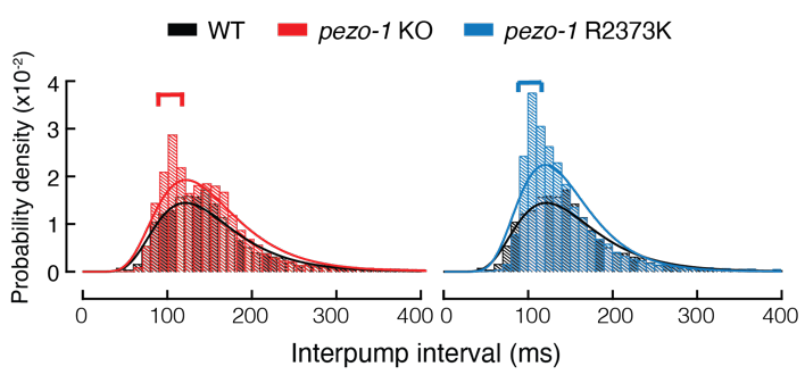

C
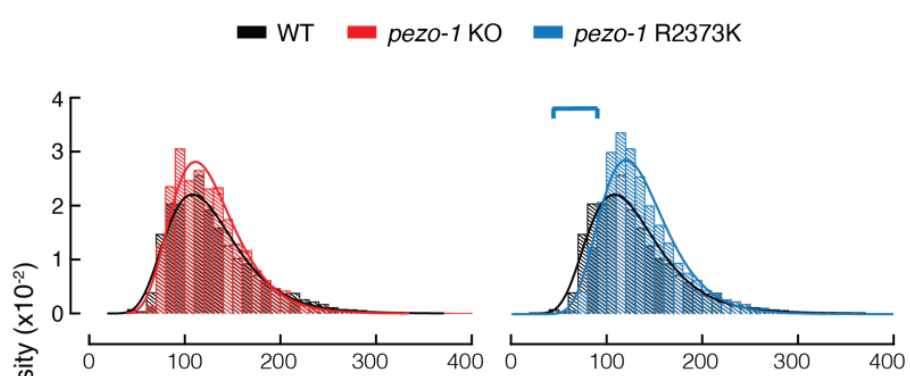

- WT $=$ eat-4 (ad572) avr-15 (ad1051)

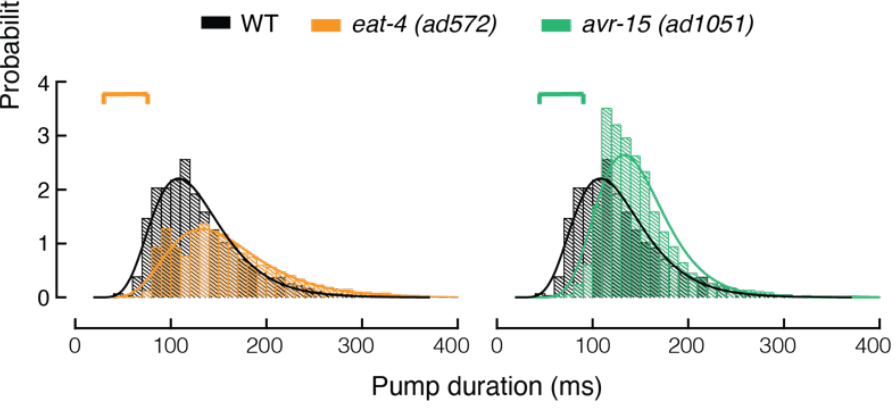

Figure 3. PEZO-1 activation affects pharyngeal pumping properties at $2 \mathrm{mM}$ serotonin. (A) pezo1 gene diagram according to wormbase.org v. WS280 made with Exon-Intron Graphic Maker WormWeb.org. Magenta rectangles and white triangles denote the 5' and 3' untranslated regions (UTR), respectively. Black rectangles denote exons and black lines denote introns. The red bracket denotes the knu508 allele (a $6,616 \mathrm{bp}$ deletion) of the pezo- $1 \mathrm{KO}$ strain and the blue triangle denotes the single point mutation (allele knu491) of the pezo-1 R2373K strain. (B) Pharyngeal pumping frequencies depicted as violin plots with the means shown as horizontal bars for WT (N2), pezo-1 KO, pezo-1 R2373K, eat-4 (ad572), and avr-15 (ad1051) strains. $\mathrm{n}$ is denoted above the $x$-axis. KruskalWallis and Dunn's multiple comparisons tests. Asterisks indicate values significantly different $(* * * \mathrm{p}<0.001)$ and ns indicates not significant. (C) Comparison between WT and pezo-1 KO, pezo-1 R2373K, eat-4 (ad572), and avr-15 (ad1051) strains pump duration distribution histograms. Smooth curves were fit to the data according to a log-normal distribution. Number of events $\geq 20,860$; bin size $30 . \mathrm{n}$ is 90 worms per condition. (D) Comparison between $\mathrm{WT}$, pezo- $1 \mathrm{KO}$, and pezo- $1 \mathrm{R} 2373 \mathrm{~K}$ strains interpump interval distribution histograms, at $2 \mathrm{mM}$ serotonin concentration. Smooth curves were fit to the data according to a log-normal distribution. Number of events $\geq 10,200$; bin size 30 . $n$ is 90 worms per condition. 
Mechanical stimuli come in many forms including stretching, bending, and osmotic forces

331 (Cox, Bavi and Martinac, 2019). To further understand the functional role of pezo-1, we evaluated

332 pharyngeal pumping parameters after challenging worm strains with different osmolarities. To this

333 end, we diluted the standard solution used for worm experiments (M9 buffer) to different osmolarities

$334(150,260$, and $320 \mathrm{mOsm})$. Low osmolarity solutions would be equivalent to swallowing food 335 containing few solutes $(150 \mathrm{mOsm})$ whereas high osmolarities would represent a "gulp" with large 336 amounts of solutes (320 mOsm). Noteworthy, the higher the osmolarity, the shorter the mean pumping 337 frequency of WT worms (Figure 4A). Our results indicate that the larger the number of solutes in 338 solution, the longer they would reside in the pharynx before moving to the intestine. Notably, at 320 $339 \mathrm{mOsm}$, both pezo- $1 \mathrm{KO}$ and GOF mutants displayed a significantly higher frequency than WT (Figure 340 4A). On the other hand, at 260 and 150 mOsm we did not measure significant differences between WT 341 and the pezo-1 mutants. Similar to human Piezo2 KO and GOF mutations (associated with joint 342 contractures), we demonstrated that lack of or enhanced PEZO-1 function modulated pharyngeal 343 pumping frequencies in the same fashion (at high osmolarities). Next, we further examined the EPG 344 parameters at high osmolarity $(320 \mathrm{mOsm})$. Analyses of the distribution of pump durations and length 345 of the mean interpump intervals revealed that both pezo- 1 mutants had more frequent fast pumps (80$346120 \mathrm{~ms}$, Figure 4B, gray rectangles) and spent less time resting between pumps than the WT (Figure 347 4C). Interestingly, high osmolarity (320 mOsm) revealed a close resemblance between PEZO-1 GOF 348 and the avr-15(ad1051) mutants' pharyngeal pumping parameters (frequency and duration, Figure 4D349 E) suggesting a potential link between PEZO-1 and $\mathrm{M} 3_{\mathrm{L} / \mathrm{R}}$ function. Altogether, our results suggest 350 that PEZO-1 is required for fine tuning pharyngeal function in response to osmolarity changes. 
A
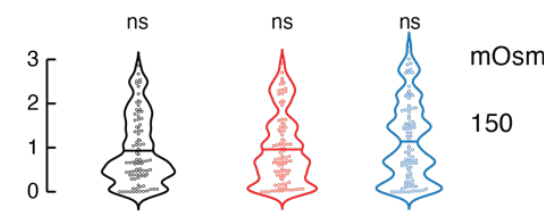

353

354

355

356

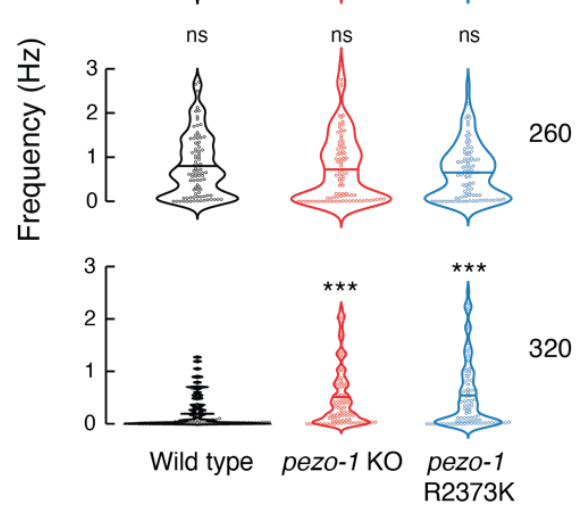

C Mean interpump interval at 320 mOsm

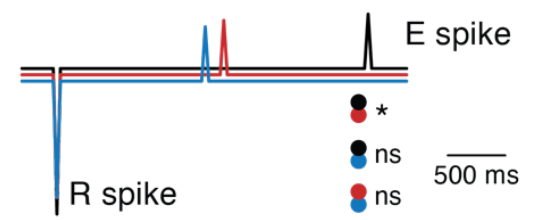

D

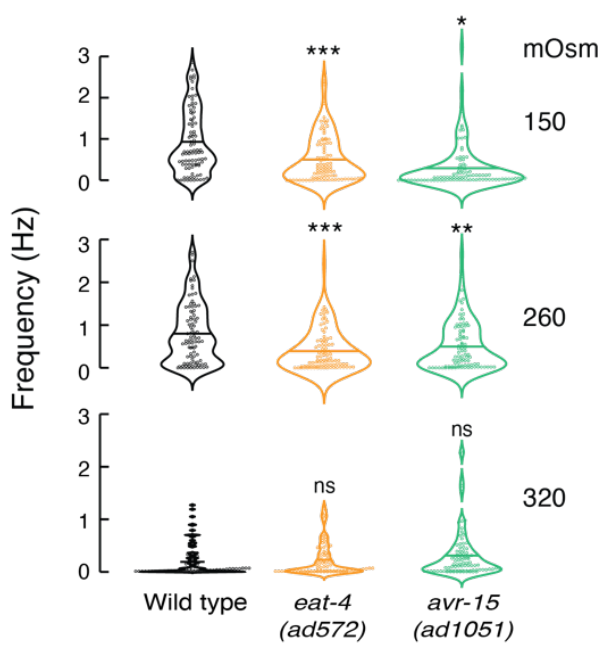

B

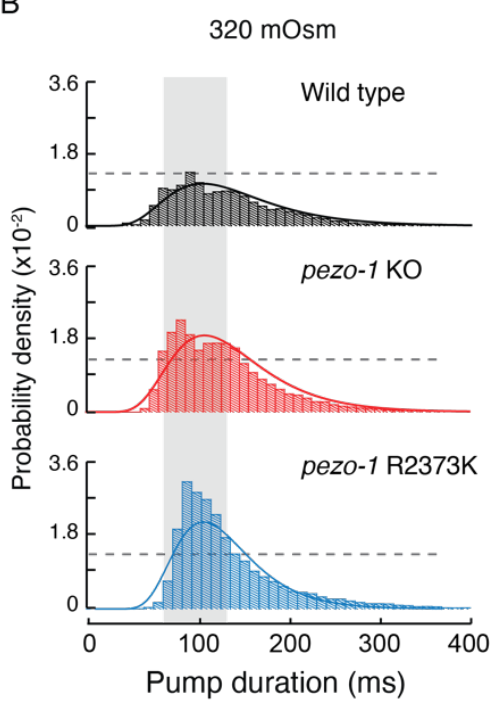

$\mathrm{E}$

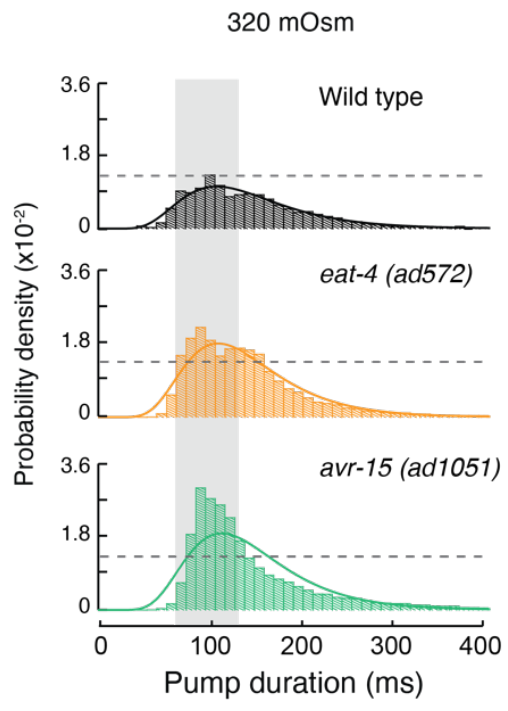

Figure 4. Osmolarity changes modify pharyngeal pumping in WT and pezo-1 strains. (A) Pharyngeal pumping frequencies depicted as violin plots with the means shown as horizontal bars for WT (N2), pezo-1 KO, and pezo-1 R2373K strains at 150, 260, and $320 \mathrm{mOsm}$. $\mathrm{n}$ is 90 worms per condition. Kruskal-Wallis and Dunn's multiple comparisons tests. (B) Comparison of probability densities for pharyngeal pumping duration of WT, pezo-1 KO, and pezo-1 R2373K strains at 150, 260, and $320 \mathrm{mOsm}$. Smooth curves were fit to the data according to a log-normal distribution. Number of events $\geq 21,110$; bin size 30. $\mathrm{n}$ is 90 worms per condition. (C) Ensemble averages of EPG traces for WT (N2), pezo-1 KO, and pezo-1 R2373K strains showing pump durations (E to R spikes) at 320 mOsm. One-way ANOVA and Tukey-Kramer multiple comparisons test. $\mathrm{n}$ is 90 worms per condition. (D) Pharyngeal pumping frequencies depicted as violin plots with the means shown as horizontal bars for WT (N2), eat-4 (ad572), and avr-15 (ad1051) strains at 150, 260, and $320 \mathrm{mOsm} . \mathrm{n}=90$, per condition. Kruskal-Wallis and Dunn's multiple comparisons tests. (E) Comparison of probability densities for pharyngeal pumping duration of WT, eat-4 (ad572), and avr-15 (ad1051) strains at 150, 260, and 320 mOsm. Smooth curves were fit to the data according to a log-normal distribution. Number of events $\geq$ 21,110; bin size $30 . \mathrm{n}$ is 90 worms per condition. Asterisks indicate values significantly different $(* * * \mathrm{p}<0.001, * * \mathrm{p}<0.01$, and $* \mathrm{p}<0.05)$ and ns indicates not significant. 


\section{PEZO-1 function is involved in food sensation}

To determine the impact that PEZO-1 function has on food intake, we recorded pharyngeal

pumping of WT and pezo-1 strains in response to different food stimuli. It has been hypothesized that

food quality and feeding preferences displayed by worms is linked to the bacteria size (Shtonda and

Avery, 2006). To this end, we measured worms pharyngeal pumping while feeding them the

conventional food used in the laboratory for maintenance (Escherichia coli strain OP50). Additionally,

we varied the dimensions of OP50 using the antibiotic cephalexin; an antibiotic that prevents the

separation of budding bacteria, generating long spaghetti-like filaments of bacterium as observed

under a microscope and elsewhere (Martinac et al., 1987) (Supplementary Figure 2A). A similar

method was previously described using the antibiotic aztreonam and has been shown to affect

pharyngeal pumping (Gruninger, Gualberto and Garcia, 2008; ben Arous, Laffont and Chatenay, 2009).

WT and pezo- 1 mutants are able to ingest spaghetti-like bacteria and reached adulthood in three

days, similar to worms fed with control bacteria (Supplementary Figure 2B-C). Notably, feeding

worms with control or spaghetti-like bacteria revealed different pharyngeal traits between the pezo-1

mutants and the WT worms. When fed with control E. coli, both pezo-1 mutants (KO and GOF) have

higher mean frequencies, shorter mean pump durations, narrower pump duration distributions, and fastest mean interpump intervals than the WT worms (Figure 5A-C, E-F). On the other hand, feeding worms with spaghetti-like $E$. coli elicits opposite effects on the pezo- 1 mutants pharyngeal pumping parameters. For instance, spaghetti-like E. coli decreases pezo-1 KO mean frequency, while keeping the mean pump duration and distribution similar to WT (Figure 5A-B, D). Furthermore, this modified diet significantly increases the mean interpump interval of the KO in comparison to the WT and the GOF mutant (Figure 5E-F). Unlike the $\mathrm{KO}$ and WT, the R2372K pezo-1 mutant displays high frequency, short pumps (mean and distributions; Figure 5A-B, D) and short mean interpump interval durations (mean and distributions; Figure 5E-F). Altogether, our results indicate that PEZO-1 regulates the pharynx response to food physical parameters, such as length and shape of the ingested bacteria. 
A

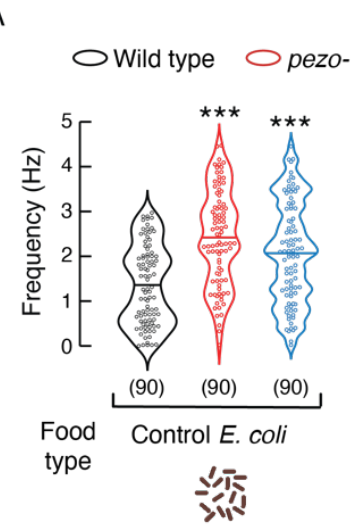

D

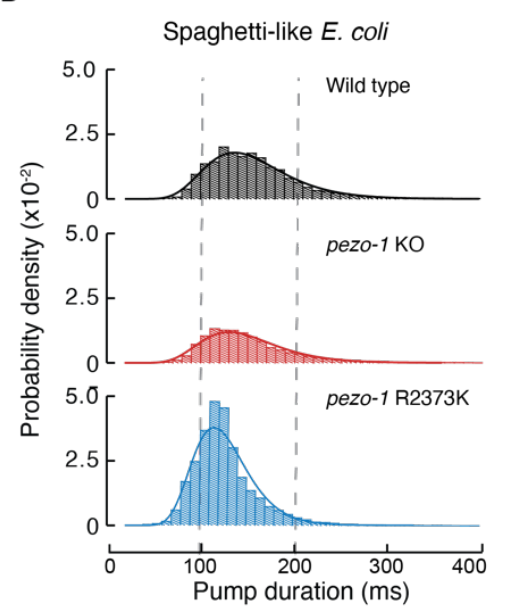

B

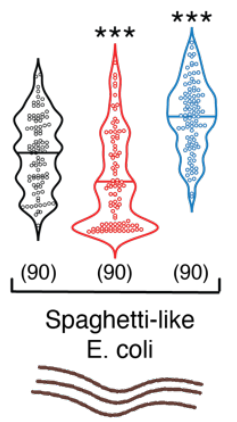

E

Mean interpump interval

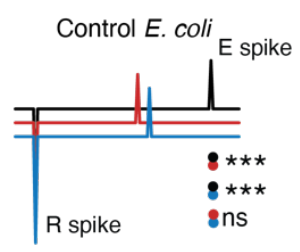

Spaghetti-like E. coli

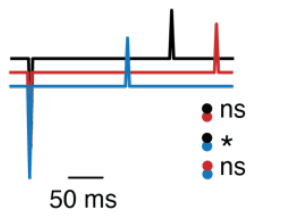

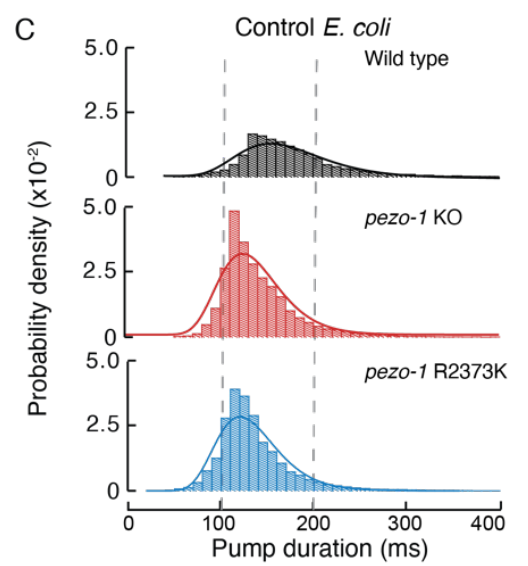

$\mathrm{F}$

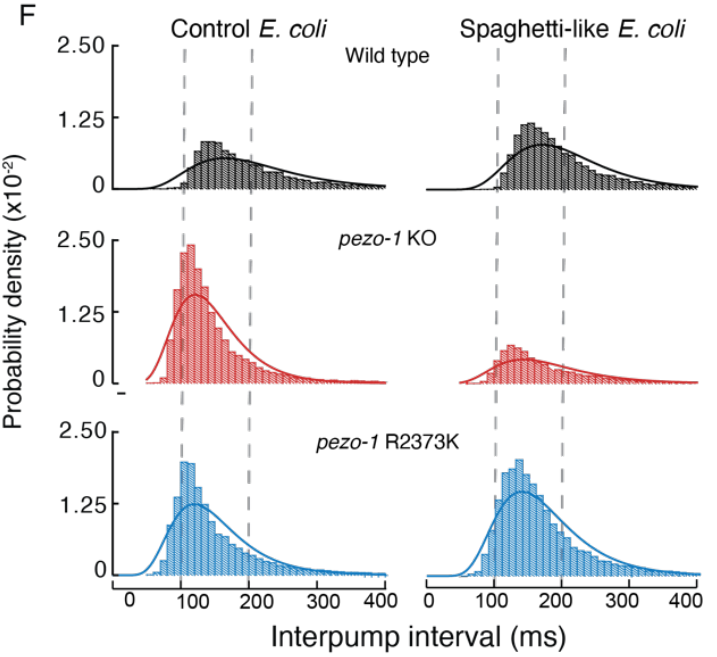

Figure 5. PEZO-1 alters pharyngeal pumping when exposed to control or spaghetti-like bacteria. (A) Pharyngeal pumping frequencies depicted as violin plots with the means shown as horizontal bars for WT (N2), pezo-1 KO, and pezo-1 R2373K when fed with control and cephalexin-treated bacteria (spaghetti-like E. coli) (bacteria cartoons were created with BioRender.com). $\mathrm{n}$ is denoted above the $x$ axis. Kruskal-Wallis and Dunn's multiple comparisons tests. Asterisks indicate values significantly different $(* * * \mathrm{p}<0.001)$. (B) Ensemble averages of EPG traces for WT (N2), pezo-1 KO, and pezo-1 R2373K strains showing pump durations (E to R spikes), when fed with control and cephalexin-treated bacteria (spaghetti-like E. coli). One-way ANOVA and Tukey-Kramer multiple comparisons test. $\mathrm{n}$ is 90 worms per condition. (C) Comparison of probability densities for pharyngeal pump duration of wild-type (N2), pezo-1 KO, and pezo-1 R2373K when fed with control bacteria. Smooth curves were fit to the data according to a log-normal distribution. Number of events $\geq 14,740$; bin size 30 . $\mathrm{n}$ is 90 worms per condition. (D) Comparison of probability densities for pharyngeal pump durations of WT (N2), pezo-1 $\mathrm{KO}$, and pezo- $1 \mathrm{R} 2373 \mathrm{~K}$ when fed with cephalexin-treated bacteria (spaghetti-like $E$. coli). Smooth curves were fit to the data according to a log-normal distribution. Number of events $\geq 19,160$; bin size 30 . $\mathrm{n}$ is 90 worms per condition. (E) Ensemble averages of EPG traces for WT (N2), pezo-1 KO, and pezo-1 R2373K strains showing interpump interval ( $\mathrm{R}$ to E spikes), when fed with control and cephalexin-treated bacteria. One-way ANOVA and Tukey-Kramer multiple comparisons test. $\mathrm{n}$ is 90 worms per condition. (F) Comparison of probability densities for pharyngeal interpump interval of WT (N2), pezo-1 KO, and pezo$1 \mathrm{R} 2373 \mathrm{~K}$ when fed with control and cephalexin-treated bacteria. Smooth curves were fit to the data according to a log-normal distribution. Number of events $\geq 9,950$; bin size $30 . \mathrm{n}$ is 90 worms per condition. Asterisks indicate values significantly different $(* * * p<0.001, * * p<0.01$, and $* \mathrm{p}<0.05)$ and ns indicates not significantly different. 


\section{pezo-1 encodes a mechanosensitive ion channel}

The PEZO-1 protein sequence shares $60-70 \%$ similarity with mammalian PIEZO channel

orthologs. However, whether PEZO-1 responds to mechanical stimuli has not yet been established. To

address this major question, we generated three different pezo- 1 strains expressing the pezo-1::GFP,

pezo- $1:: \mathrm{GFP} \mathrm{KO}$, and pezo-1::GFP R2373K mutation. pezo-1::GFP cells were patch-clamped using

the cell-attached configuration while applying constant negative pressure $(-70 \mathrm{mmHg})$ and steps of

positive and negative voltages to the pipette (Figure 6A-C). The current vs. voltage relationship is

slight cation selective conductance like the mouse and Drosophila's orthologs (Coste et al., 2012).

A

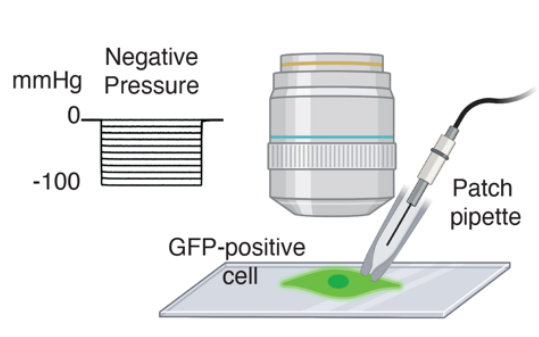

C

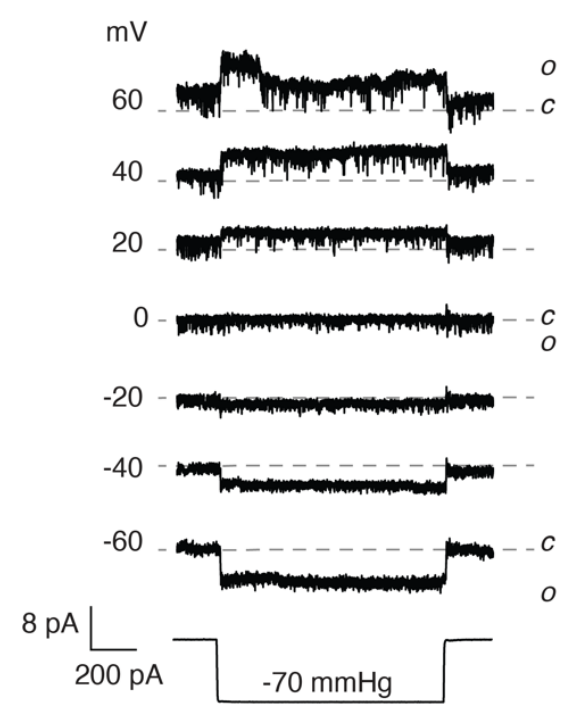

B

C. elegans embryo cultures
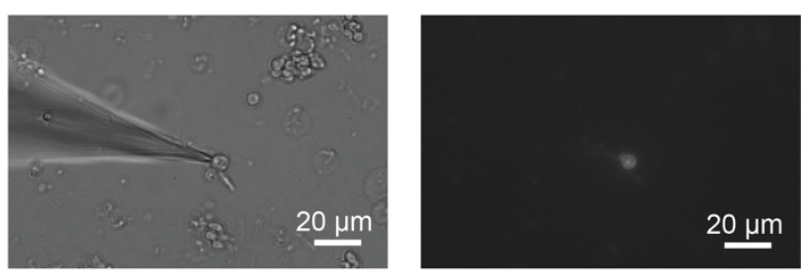

D

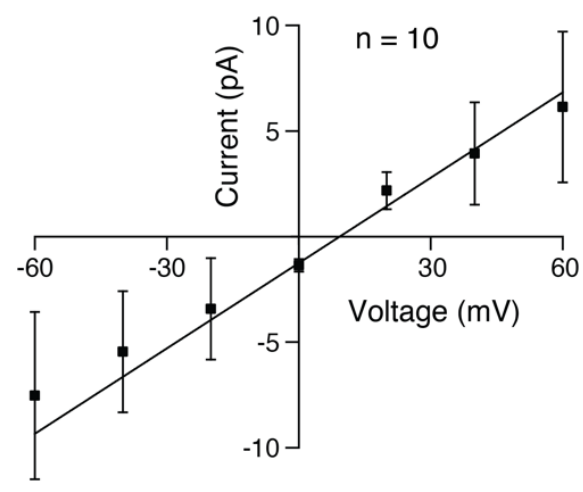

Figure 6. Cells expressing PEZO-1 display mechanosensitive channel currents (A) Schematic representation of the mechanical stimulation protocol applied to pezo-1::GFP expressing cells recorded in the cell-attached configuration. Created with BioRender.com. (B) Representative micrographs (from at least 10 independent preparations) of a C. elegans primary embryonic culture from pezo- $1:$ GFP expressing strains. Brightfield (left) and fluorescence (right) micrographs of a pezo-1::GFP expressing cell when patch clamped in the on-cell configuration. (C) Representative cell-attached patch-clamp recordings of mechanically-activated currents from WT, pezo-1 KO, and pezo-1 R2373K cells expressing pezo-1::GFP. Channel openings (downward) were elicited by -70 $\mathrm{mmHg}$ square pulse (bottom) at constant voltages ranging from -60 to $+60 \mathrm{mV}$. Gray dashed line represents background currents. Closed and open states are labeled $c$ and $o$, respectively. (D) Current-voltage relation recorded at a constant pressure of $-70 \mathrm{mmHg}$ in the on-cell configuration. The reversal potential is $9.31 \mathrm{mV}$. Each circle represents the mean $\pm \mathrm{SD}$. 
(Figure 7A-C, black traces and bar) with a half pressure activation $\left(\mathrm{P}_{1 / 2}\right)$ corresponding to $-59.1 \pm 4.3$

$\mathrm{mmHg}$ (mean $\pm \mathrm{SEM}$ ). Importantly, pezo-1::GFP cells expressing KO PEZO-1 did not feature mechanosensitive currents, even at larger negative pressure magnitudes (Figure 7A and C, red traces and bar). On the other hand, PEZO-1 R2373K displayed mechano-dependent currents (Figure 7A-C, blue traces and bar) with lower $\mathrm{P}_{1 / 2}$ than the WT channel $(39.2 \pm 2.2 \mathrm{mmHg}$, mean $\pm \mathrm{SEM})$, indicating that the GOF mutant requires less mechanical activation to open. Notably, the R2373K mutation introduced a latency for activation that was not detected in the WT (Figure 7A, blue traces and 7D). The decrease in mechanical threshold along with the slow activation were previously reported for the equivalent human PIEZO1 R2456K mutation in mammalian cell lines (Zarychanski et al., 2012; Albuisson et al., 2013; Bae et al., 2013; Romero et al., 2019). Future experiments are needed to understand the origin of these differences in activation. Unlike pezo- $1 \mathrm{WT}$, approximately $50 \%$ of the mechano currents elicited from the pezo-1 R2373K expressing cells remained active even after the mechanical stimulus ended (Figure 7A, blue traces, and 7E). This slow deactivation is also reminiscent of the human PIEZO1 R2456K GOF phenotype previously characterized by Bae and collaborators (Bae et al., 2013). Overall, our results support that PEZO-1 is an ion channel gated by membrane tension and that a conservative mutation in the pore domain elicits similar activation and kinetic changes as its human counterpart.

To further validate that the pezo- 1 gene encodes for a mechanosensitive ion channel, we heterologously expressed one of the longest isoforms of pezo-1 (isoform G; wormbase.org v. WS280) in Sf9 cells. Similar to mammalian PIEZO channels, PEZO-1 mediates indentation-activated currents (Figure 8). Uninfected Sf9 cells do not display mechanosensitive channel currents (Figure 8B-C). Importantly PEZO-1 displayed the properties described for mammalian PIEZOs in other cell types including (Coste et al., 2010; Wu et al., 2017) non-selective cation currents, as determined by the reversal potential $(+6.74 \mathrm{mV}$; Figure 8D-E) and voltage-dependent inactivation (Figure 8D and 8F). 
A

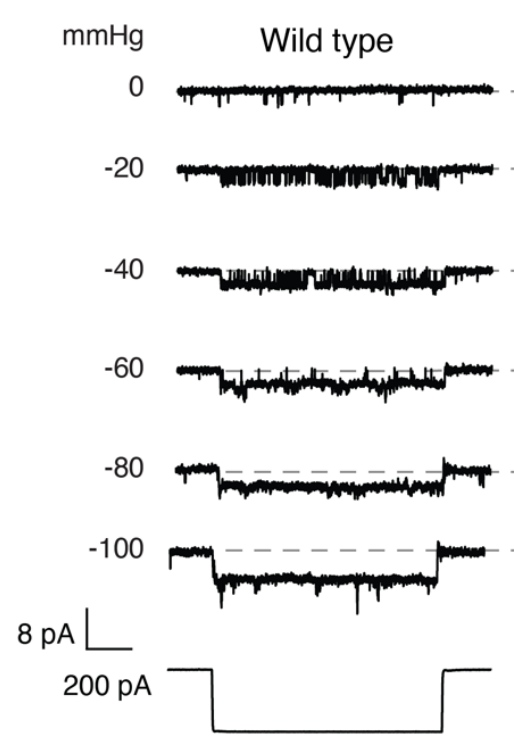

B

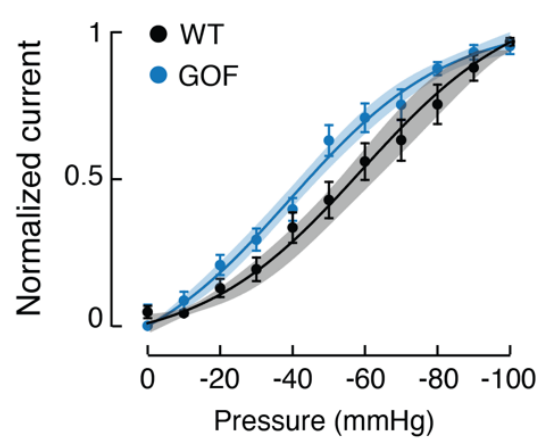

pezo-1 KO pezo-1 R2373K

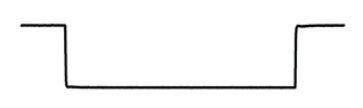

C

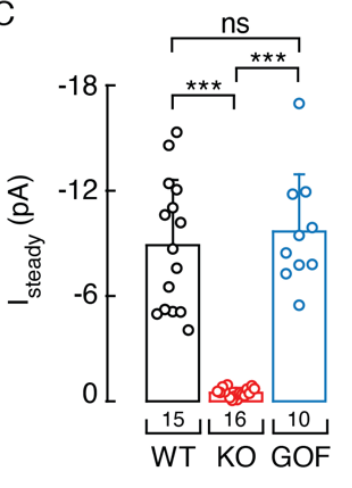

D

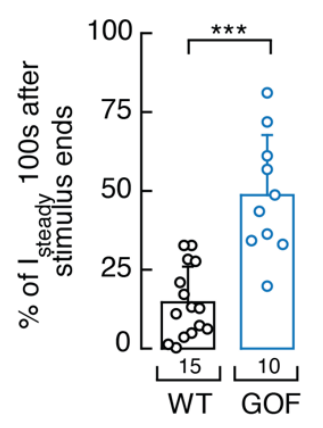

$\mathrm{E}$

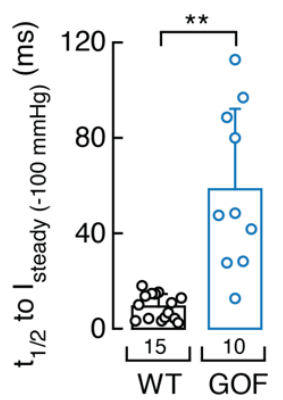

Figure 7. PEZO-1 GOF mutant decreases mechanical threshold and slows down deactivation. (A) Representative cell-attached patch-clamp recordings of mechanically-activated currents from WT, pezo-1 KO, and pezo-1 R2373K cells expressing pezo-1::GFP. Channel openings (downward) were elicited by negative pressure (left) square pulses (bottom) at a constant voltage of $-60 \mathrm{mV}$. Gray dashed line represents background currents and channel openings are downward. (B) Pressure-response profiles for PEZO-1 WT and R2373K currents. Normalized currents elicited by negative pressure of mechanically activated currents of WT and pezo-1 R2373K cells expressing pezo-1::GFP. A Boltzmann function, Eq. (1), was fitted to the data. The shadows below the curves indicate the $95 \%$ confidence bands for the fit. Circles are mean \pm SD. $\mathrm{n}$ for WT and pezo-1 R2373K are 15 and 10, respectively. (C) Bar graph displaying steady state currents elicited by $-100 \mathrm{mmHg}$ of negative pressure of WT, pezo-1 KO, and pezo-1 R2373K cells expressing pezo-1: GFP. Bars are mean \pm SD. $\mathrm{n}$ is denoted above the $x$-axis. Kruskall-Wallis and Dunn's multiple comparisons tests. (D) Bar graph displaying the time it takes to reach half of the steady state currents elicited with $-100 \mathrm{mmHg}$ of pressure of $\mathrm{WT}$ and pezo-1 $\mathrm{R} 2373 \mathrm{~K}$ cells expressing pezo-1::GFP. Bars are all mean $\pm \mathrm{SD}$. $\mathrm{n}$ is denoted above the $x$-axis. Unpaired $t$-test with Welch's correction. (E) Bar graph displaying percentage of steady state currents left $100 \mathrm{~ms}$ after the mechanical stimulus ended of WT and pezo-1 R2373K cells expressing pezo-1::GFP. Bars are mean $\pm \mathrm{SD} . \mathrm{n}$ is denoted above the $x$-axis. Unpaired $t$-test. Asterisks $(* * * \mathrm{p}<0.001$ and $* * \mathrm{p}<0.01)$ indicate values significantly different. ns indicates not significant. 
A

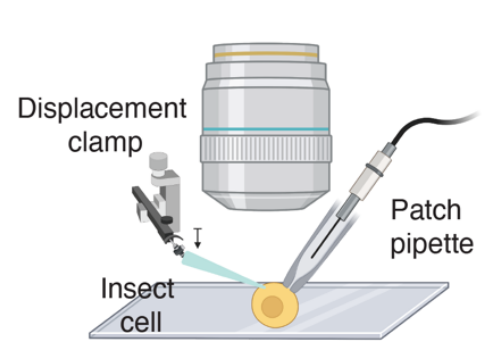

D

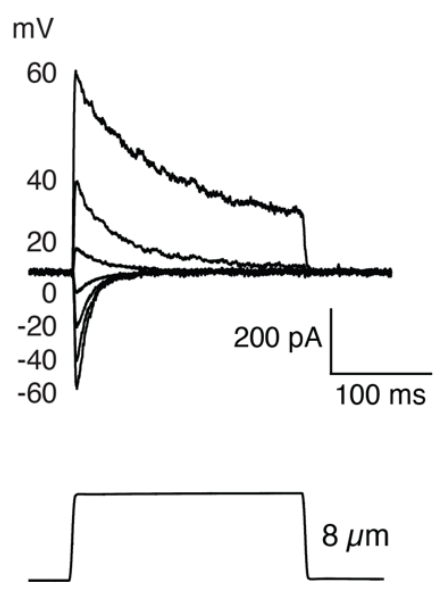

B

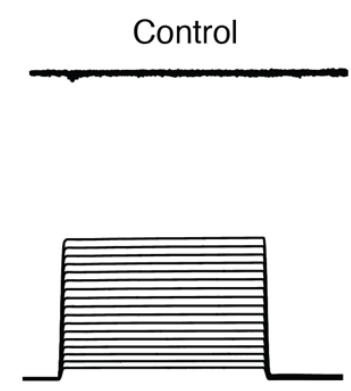

E
SF9 cells

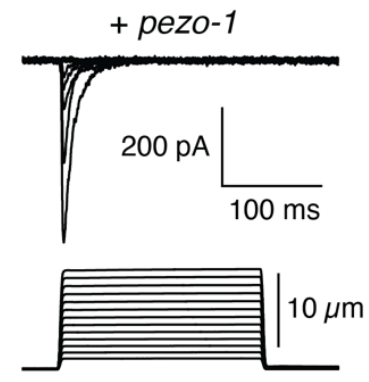

C

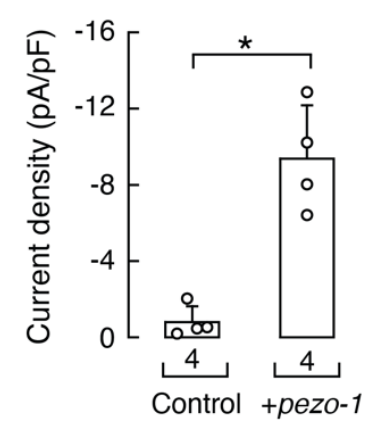

$\mathrm{F}$
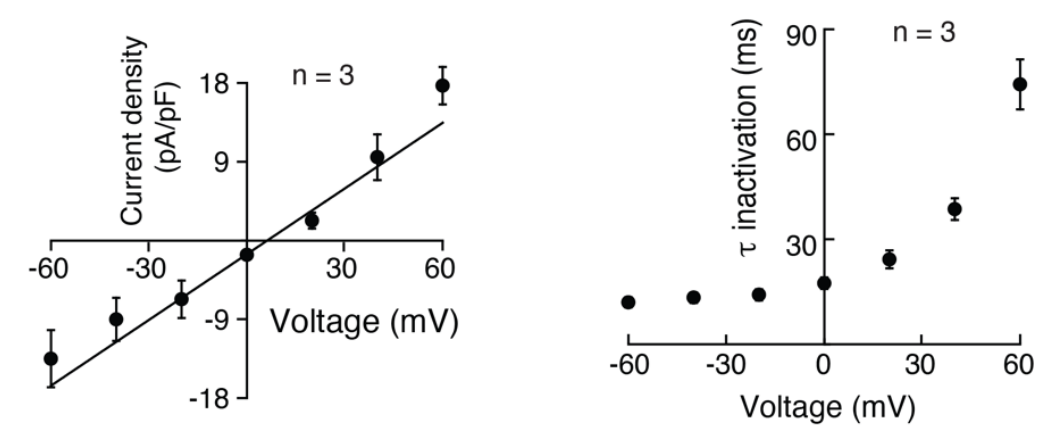

Figure 8. Sf9 cells infected with a pezo-1-containing baculovirus display mechanosensitive currents. (A) Schematic representation of the mechanical stimulation protocol applied to Sf9 cells infected with a baculovirus containing pezo-1 recorded in the whole-cell configuration. Created with BioRender.com. (B) Representative whole-cell patch-clamp recordings (at $-60 \mathrm{mV}$ ) of Sf9 cells control or infected with a baculovirus containing pezo-1 elicited by mechanical stimulation. (C) Current densities of Sf9 cells control or infected with a baculovirus containing pezo-1 elicited by maximum displacement. $\mathrm{n}$ is denoted above the $x$-axis. Mann-Whitney test. Asterisk indicates values significantly different $(* \mathrm{p}<0.05)$. (D) Representative whole-cell patch-clamp recordings of Sf9 cells infected with a baculovirus containing pezo- 1 elicited by mechanical stimulation (bottom) at constant voltages ranging from -60 to $+60 \mathrm{mV}$. (E) Current-voltage relation recorded at a constant displacement of $8 \mu \mathrm{m}$ in the whole-cell configuration. The reversal potential is $6.74 \mathrm{mV}$. Each circle represents the mean $\pm \mathrm{SD}$. (F) PEZO-1 time constants of inactivation elicited by maximum displacement at constant voltages ranging from -60 to $+60 \mathrm{mV}$. Each circle represents the mean $\pm \mathrm{SD}$. 


\section{DISCUSSION}

In 2010, Coste and collaborators reported that the C. elegans genome contained a single Piezo

440 gene, pezo-1 (Coste et al., 2010). However, the functional role of pezo-1 remained elusive even after

441 a decade of its discovery. Here, we showed that PEZO-1 is a mechanosensitive channel with a novel

442 functional role in the worm pharynx by combining fluorescent reporters, genome editing,

443 electropharyngeogram, behavioral, and patch-clamp measurements. We found that pezo-1 is highly

444 expressed in neurons involved in pharyngeal pumping relaxation. In addition to its expression, several

445 lines of evidence suggested that PEZO-1 modulated several discrete but reliable features of the 446 pharyngeal function. Lack- or augmentation- of PEZO-1 function increased pharyngeal pumping

447 frequencies when worms were challenged with $2 \mathrm{mM}$ serotonin, hyperosmotic conditions, or fed with 448 control bacteria. In the absence of functional PEZO-1, worms reduced pharyngeal function (i.e., low 449 frequency and long pump intervals) when fed with spaghetti-like bacteria. Finally, we demonstrated 450 that the pezo-1 gene encodes a mechanosensitive ion channel. Altogether, our results established that 451 PEZO-1 is important for pharyngeal function regulation and food sensation.

tube of electrically coupled muscle cells that continuously pump throughout the worm's life (Mango,

2007). Several ion channels have been identified to be crucial for the pharyngeal muscle action potential, including acetylcholine receptors, T- and L-type $\mathrm{Ca}^{2+}$ channels, glycine receptors, and $\mathrm{K}^{+}$ channels (Avery and You, 2012). Although the pharyngeal muscle is capable of pumping (albeit at low frequencies) without nervous system input, higher pumping frequencies are controlled by pharyngeal motor neurons, namely $\mathrm{MC}_{\mathrm{L} / \mathrm{R}}$ and $\mathrm{M} 3_{\mathrm{L} / \mathrm{R}}$ (Avery and You, 2012). Nevertheless, the role of the nervous system in the control of rhythmic pharyngeal pumping is not completely understood. It is 460 known, however, that the pharynx responds to a variety of neuromodulators (Avery and Horvitz, 1989). We found that pezo- 1 is expressed in proprioceptive/mechanosensory neurons $\mathrm{NSM}_{\mathrm{L} / \mathrm{R}}$ and

$462 \mathrm{M} 3_{\mathrm{L} / \mathrm{R}}$ (both important for the pharyngeal nervous system) and in the pharyngeal interneuron I3 (Avery 
1993; Avery and Thomas, 1997). Our results suggest that PEZO-1 is not essential for pharyngeal muscles, but fine tunes the role of the nervous system controlling the pharynx function. This is reminiscent of the novel role of mammalian PIEZO1 and PIEZO2 mediating neuronal sensing of blood pressure and the baroreceptor reflex (Zeng et al., 2018).

$\mathrm{NSM}_{\mathrm{L} / \mathrm{R}}$ and $\mathrm{M} 3_{\mathrm{L} / \mathrm{R}}$, both pezo-1-expressing neurons, have been postulated to sense bacteria in 469 the pharynx lumen via their proprioceptive endings and secrete serotonin in response to this mechanical stimulus (Avery, 1993; Avery and Thomas, 1997). Laser ablation of $\mathrm{NSM}_{\mathrm{L} / \mathrm{R}}$ in unc-29 mutants leads to subtle changes in pharyngeal pumping rate; however, this was done while simultaneously ablating other pharyngeal motor neurons $\left(\mathrm{M} 1, \mathrm{M} 2_{\mathrm{L} / \mathrm{R}}, \mathrm{M} 3_{\mathrm{L} / \mathrm{R}}, \mathrm{M} 5\right.$, and $\mathrm{MI}$ ) (Avery, 1993). This approach could exert antagonistic effects on pumping rate yielding a steady pharyngeal activity. Using the Screenchip ${ }^{\mathrm{TM}}$ system allowed us to reveal the potential roles of extrapharyngeal neurons expressing pezo-1 $\left(\mathrm{NSM}_{\mathrm{L} / \mathrm{R}}\right.$ and $\left.\mathrm{M}_{\mathrm{L} / \mathrm{R}}\right)$. Our results determined that activation of PEZO-1 inhibited serotonin-dependent fast pumping rate in the absence of food. They further demonstrated that PEZO-1 modulated the feeding behavior of worms confronted to various food consistencies (control and spaghetti-like bacteria). This led us to hypothesize that PEZO-1 is involved in food sensation and modulates pharyngeal pumping rate. Hence, similar to the mammalian ortholog PIEZO2, PEZO-1 is expressed in proprioceptive endings and involved in stretch reflexes (Woo et al., 2015; Chesler et al., 2016). Nevertheless, it remains to be determined if mammalian PIEZO channels play a role in food sensation and/or the swallowing reflex.

Humans sense various organoleptic food qualities such as visual aspects (color and shape), odorants through smell, and texture and flavor through tasting. In nematodes, there is a lack of understanding of what is sensed as food. Worms are able to filter particles from fluid in a sizedependent manner (Fang-Yen, Avery and Samuel, 2009; Kiyama, Miyahara and Ohshima, 2012) and feeding is facilitated by attractive odors or suppressed by repellents (e.g., diacetyl, isoamyl alcohol, quinine) (Gruninger, Gualberto and Garcia, 2008; Li et al., 2012). Others have demonstrated that 
490 rather than inactive bacteria (Yu et al., 2015). We determined that pezo- $1 \mathrm{KO}$ worms "choke" when

491 presented with spaghetti-like bacteria, whereas WT and GOF strains increase pharyngeal pumping

492 when ingesting this elongated food. Therefore, we propose that the pharynx itself might be a sensory

493 organ, as worms modify their pumping parameters when they sense solutions of different osmolarities

494 or food with different textures and/or consistencies. We further hypothesized that worms are able to

495 perceive changes in texture and adjust their pumping frequency by a mechanism requiring PEZO-1.

496 Since pezo-1 is not essential for C. elegans when cultured in standard laboratory conditions (e.g., 497 monoaxenically on E. coli OP50), we wonder if in its natural biotic environment this mechanosensitive 498 ion channel plays a crucial role, as it does in humans and Drosophila. Given that worms grow in 499 microbe-rich and heterogenous environments (feeding from prokaryotes of the genera Acetobacter, 500 Gluconobacter, and Enterobacter) (Schulenburg and Félix, 2017), they might encounter bacteria of 501 different dimensions and textures that will make pezo- 1 function more relevant to the worm's ability 502 to discriminate the food on which it grows best.

Why pezo-1 loss- and gain-of-function mutations cause similar behavior phenotypes? Our data

504 show that both pezo-1 mutants (KO and GOF) increase the pumping frequency of the pharynx in 505 different settings: serotonin exposure, high osmolarity, and ingestion of control bacteria. While it may 506 seem counterintuitive at first, there are several scenarios in which too little or too much 507 mechanosensation can be detrimental for animal's behaviors. For instance, the mec-4 gene encodes 508 for the $\mathrm{DEG} / \mathrm{ENaC}$ ion channel subunit of the mechanoelectrical transduction channel complex in 509 touch. Like our pezo-1 mutants that lack control of pharyngeal pumping function, LOF (u253, a 510 deletion) and GOF (e1611, missense mutation A713V) alleles of the mec-4 gene render touch511 insensitive worms (Driscoll and Chalfie, 1991; Hong, Mano and Driscoll, 2000; O’Hagan, Chalfie and 512 Goodman, 2005). Similarly, deg-1, a DEG/ENaC ion channel subunit expressed in ASH neurons, LOF 513 (u443, eliminates the 3' end of deg-1) and GOF (u506, missense mutation A393T) alleles decrease the 514 worm's ability to respond to nose touch (Savage et al., 1989; García-Añoveros, Ma and Chalfie, 1995; 515 Geffeney et al., 2011). In humans, PIEZO2 LOF (premature stop codon) and GOF (missense mutation 
I802F) alleles caused joint contractures, skeletal abnormalities and alterations in muscle tone (Coste et al., 2013; Chesler et al., 2016; Yamaguchi et al., 2019). Only when feeding worms, the spaghetti-

518 like bacteria, we were able to uncover a difference between the LOF and the GOF mutants. Hence, we 519 hypothesize that lacking the function of PEZO-1 significantly slows down pharyngeal function when passing down the lengthy bacteria from the pharynx to the gut.

Several requirements must be met for a channel to be considered mechanically gated (Arnadóttir and Chalfie, 2010). Accordingly, we found that pezo- 1 is expressed in the proprioceptive neuron NSM, knocking out pezo-1 inhibits worm's pharyngeal function when challenged with hyperosmolarity or elongated bacteria, engineering a single point mutation in the putative pore domain (R2373K) elicited similar activation and deactivation delays that are reminiscent of the gating behavior reported for the human PIEZO1 R2456K (Bae et al., 2013), and expression of pezo-1 confers mechanosensitivity to, otherwise naïve, Sf9 cells. We propose that PEZO-1 is a mechanosensitive ion channel directly gated by bilayer tension given that the time it takes to reach half of the steady state currents ranges between 3.5 to $15 \mathrm{~ms}$ upon application of negative pressure. These are faster than activation times reported for the Drosophila phototransduction cascade, one of the quickest second messenger cascades (Hardie, 2001). These combined efforts highlight the versatile functions of the PIEZO mechanosensitive channel family as well as the strength of the model organism C. elegans to reveal physiological functions.

Our findings revealing PEZO-1 as a mechanosensitive ion channel that modulates pharyngeal function raise several important questions. How does pezo-1 modulate pumping behavior electrical activity? Does pezo- 1 equally enhance or inhibit the function of the pharyngeal hypodermal, gland and muscle cells, and neurons expressing this channel? Could pezo- 1 phenotypes be exacerbated if the gene function is nulled in a cell-specific manner? Does PEZO-1 require auxiliary subunits and/or cytoskeleton for gating? Regardless of the answers, the plethora of physiological roles that this eukaryotic family of mechanosensitive ion channels play is outstanding. More experimental insight 


\section{Acknowledgements}

The authors thank Dr. Julio F. Cordero-Morales, Dr. Andrés G. Vidal-Gadea, and Dr.

544 Christopher E. Hopkins for critically reading the manuscript, and Dr. Rebeca Caires, MSc Briar Bell,

545 and MBBS Soumi Mazumdar for technical assistance. C. elegans (N2, DA572, and DA1051) and E.

546 coli strains (OP50 and NA22) were obtained from the Caenorhabditis Genetics Center, which is

547 funded by the NIH Office of Research Infrastructure Programs (P40 OD010440). LX960 was provided

548 by Dr. Kevin Collins (University of Miami). This work was supported by the American Heart

549 Association (16SDG26700010 to VV) and the National Institutes of Health (R01GM133845 to VV)

550 and the Neuroscience Institute at UTHSC (Research Associate Matching Salary Support to JL).

\section{Competing interests}

The authors declare no competing financial interests.

\section{Author contributions}

554 Conceptualization, VV; Methodology, VV and JRMM; Investigation, JRMM, LOR, and JL;

555 Writing, VV and JRMM; Funding Acquisition, VV; Supervision, VV. 


\begin{tabular}{l|c}
\hline Comparison & p-value \\
\hline '0mM'vs '2mM' & $2.31 \mathrm{E}-31$ \\
\hline '0mM'vs '5mM' & $8.82 \mathrm{E}-40$ \\
\hline '0mM'vs '10mM' & $1.88 \mathrm{E}-40$ \\
\hline '0mM'vs '20mM' & $1.88 \mathrm{E}-40$ \\
\hline '2mM'vs '5mM' & $3.52 \mathrm{E}-16$ \\
\hline '2mM'vs '10mM' & $7.35 \mathrm{E}-25$ \\
\hline '2mM'vs '20mM' & $9.25 \mathrm{E}-28$ \\
\hline '5mM'vs '10mM' & 0.000142 \\
\hline '5mM'vs '20mM' & $1.77 \mathrm{~m}-06$ \\
\hline '10mM'vs '20mM' & 0.10003 \\
\hline
\end{tabular}




\section{REFERENCES}

564

565

566

567

568

569

570

571

572

573

574

575

576

577

578

579

580

581

582

583

584

585

586

587

588

589

590

591

592

593

594

595

596

597

598

599

600

601

602

603

604

605

606

607

608

609

610

611

612

Albertson, D. G. and Thomson, J. N. (1976) “The pharynx of Caenorhabditis elegans,” Philosophical Transactions of the Royal Society of London. B, Biological Sciences, 275(938), pp. 299-325. doi: 10.1098/rstb.1976.0085.

Albuisson, J. et al. (2013) "Dehydrated hereditary stomatocytosis linked to gain-of-function mutations in mechanically activated PIEZO1 ion channels," Nature Communications, 4(May). doi: $10.1038 /$ ncomms 2899 .

Alper, S. L. (2017) “Genetic Diseases of PIEZO1 and PIEZO2 Dysfunction," Current Topics in Membranes, 79. doi: 10.1016/bs.ctm.2017.01.001.

ben Arous, J., Laffont, S. and Chatenay, D. (2009) "Molecular and Sensory Basis of a Food Related Two-State Behavior in C. elegans," PLoS ONE. Edited by V. Brezina, 4(10), p. e7584. doi: 10.1371/journal.pone.0007584.

Arnadóttir, J. and Chalfie, M. (2010). "Eukaryotic mechanosensitive channels. Annu Rev Biophys., pp. 39:111-37. doi: 10.1146/annurev.biophys.37.032807.125836. PMID: 20192782.

Avery, L. (1993) "Motor neuron M3 controls pharyngeal muscle relaxation timing in Caenorhabditis elegans.," The Journal of experimental biology, 175, pp. 283-297.

Avery, L., Bargmann, C. I. and Horvitz, H. R. (1993) "The Caenorhabditis elegans unc-31 gene affects multiple nervous system-controlled functions.," Genetics, 134(2), pp. 455-64. Available at: http://www.ncbi.nlm.nih.gov/pubmed/8325482.

Avery, L. and Horvitz, H. R. (1989) "Pharyngeal pumping continues after laser killing of the pharyngeal nervous system of C. elegans," Neuron, 3(4), pp. 473-485. doi: 10.1016/08966273(89)90206-7.

Avery, L. and Thomas, J. H. (1997) "Feeding and Defecation," in. Cold Spring Harbor Laboratory Press, Cold Spring Harbor (NY). Available at: https://www.ncbi.nlm.nih.gov/books/NBK20138. Avery, L. and You, Y.-J. (2012) "C. elegans feeding.," WormBook: the online review of C. elegans biology, pp. 1-23. doi: 10.1895/wormbook.1.150.1.

Bae, C. et al. (2013) "Xerocytosis is caused by mutations that alter the kinetics of the mechanosensitive channel PIEZO1," Proceedings of the National Academy of Sciences of the United States of America, 110(12). doi: 10.1073/pnas.1219777110.

Bai, X. et al. (2020) "Caenorhabditis elegans piezo channel coordinates multiple reproductive tissues to govern ovulation," eLife, 9, pp. 1-35. doi: 10.7554/eLife.53603.

Chesler, A. T. et al. (2016) "The Role of PIEZO2 in Human Mechanosensation.," The New England journal of medicine, 375(14), pp. 1355-1364. doi: 10.1056/NEJMoa1602812.

Coste, B. et al. (2010) "Piezo1 and Piezo2 Are Essential Components of Distinct Mechanically Activated Cation Channels," Science, 330(6000), pp. 55-60. doi: 10.1126/science.1193270.

Coste, B. et al. (2012). "Piezo proteins are pore-forming subunits of mechanically activated channels". Nature, 483(7388), pp. 176-81. doi: 10.1038/nature10812.

Coste, B. et al. (2013) "Gain-of-function mutations in the mechanically activated ion channel PIEZO2 cause a subtype of Distal Arthrogryposis," Proceedings of the National Academy of Sciences of the United States of America, 110(12). doi: 10.1073/pnas.1221400110.

Cox, C. D., Bavi, N. and Martinac, B. (2019) "Biophysical Principles of Ion-Channel-Mediated Mechanosensory Transduction,” Cell Reports. doi: 10.1016/j.celrep.2019.08.075.

Dent, J. A., Davis, M. W. and Avery, L. (1997) "avr-15 encodes a chloride channel subunit that mediates inhibitory glutamatergic neurotransmission and ivermectin sensitivity in Caenorhabditis elegans," The EMBO Journal, 16(19), pp. 5867-5879. doi: 10.1093/emboj/16.19.5867.

Douguet, D. and Honoré, E. (2019) "Mammalian Mechanoelectrical Transduction: Structure and Function of Force-Gated Ion Channels.," Cell, 179(2), pp. 340-354. doi: 10.1016/j.cell.2019.08.049. Driscoll, M. and Chalfie, M. (1991) "The mec-4 gene is a member of a family of Caenorhabditis elegans genes that can mutate to induce neuronal degeneration," Nature, 349(6310), pp. 588-593. doi: $10.1038 / 349588 \mathrm{a} 0$. 
613 Fang-Yen, C., Avery, L. and Samuel, A. D. T. (2009) "Two size-selective mechanisms specifically 614 trap bacteria-sized food particles in Caenorhabditis elegans," Proceedings of the National Academy 615 of Sciences, 106(47), pp. 20093-20096. doi: 10.1073/pnas.0904036106.

616 García-Añoveros, J., Ma, C. and Chalfie, M. (1995) "Regulation of Caenorhabditis elegans 617 degenerin proteins by a putative extracellular domain," Current Biology, 5(4), pp. 441-448. doi: 618 10.1016/S0960-9822(95)00085-6.

619 Geffeney, S. L. et al. (2011) "DEG/ENaC but not TRP channels are the major mechanoelectrical 620 transduction channels in a c. Elegans nociceptor," Neuron, 71(5), pp. 845-857. doi:

$621 \quad 10.1016 /$ j.neuron.2011.06.038.

622 Geffeney, S. L. and Goodman, M. B. (2012) "How we feel: ion channel partnerships that detect mechanical inputs and give rise to touch and pain perception.," Neuron, 74(4), pp. 609-19. doi: 10.1016/j.neuron.2012.04.023.

Gruninger, T. R., Gualberto, D. G. and Garcia, L. R. (2008) "Sensory perception of food and insulinlike signals influence seizure susceptibility.," PLoS genetics, 4(7), p. e1000117. doi:

10.1371/journal.pgen.1000117.

Hamilton, E. S., Schlegel, A. M. and Haswell, E. S. (2015) "United in diversity: mechanosensitive ion channels in plants.," Annual review of plant biology, 66, pp. 113-37. doi: 10.1146/annurevarplant-043014-114700.

Hardie, R. C. (2001) "Phototransduction in Drosophila melanogaster," Journal of Experimental Biology.

Hasse, S., Hyman, A. A. and Sarov, M. (2016) "TransgeneOmics - A transgenic platform for protein localization based function exploration," Methods, 96, pp. 69-74. doi: 10.1016/j.ymeth.2015.10.005. Höflich, J. et al. (2004) "Loss of srf-3 -encoded Nucleotide Sugar Transporter Activity in C aenorhabditis elegans Alters Surface Antigenicity and Prevents Bacterial Adherence," Journal of Biological Chemistry, 279(29), pp. 30440-30448. doi: 10.1074/jbc.M402429200.

Hong, K., Mano, I. and Driscoll, M. (2000) "In vivo structure-function analyses of Caenorhabditis elegans MEC-4, a candidate mechanosensory ion channel subunit," Journal of Neuroscience, 20(7), pp. 2575-2588. doi: 10.1523/jneurosci.20-07-02575.2000.

641 Ikeda, R. et al. (2014) "Merkel cells transduce and encode tactile stimuli to drive A $\beta$-afferent impulses.," Cell, 157(3), pp. 664-75. doi: 10.1016/j.cell.2014.02.026.

Keane, J. and Avery, L. (2003) "Mechanosensory inputs influence Caenorhabditis elegans pharyngeal activity via ivermectin sensitivity genes.," Genetics, 164(1), pp. 153-62. Available at: http://www.ncbi.nlm.nih.gov/pubmed/12750328.

Kim, S. E. et al. (2012) “The role of Drosophila Piezo in mechanical nociception," Nature, 483(7388). doi: 10.1038/nature10801.

Kiyama, Y., Miyahara, K. and Ohshima, Y. (2012) "Active uptake of artificial particles in the nematode Caenorhabditis elegans," Journal of Experimental Biology, 215(7), pp. 1178-1183. doi: 10.1242/jeb.067199.

651 Kung, C., Martinac, B. and Sukharev, S. (2010) "Mechanosensitive channels in microbes.," Annual review of microbiology, 64, pp. 313-29. doi: 10.1146/annurev.micro.112408.134106.

Lee, K. S. et al. (2017) "Serotonin-dependent kinetics of feeding bursts underlie a graded response to food availability in C. elegans," Nature Communications, 8(1), p. 14221. doi:

$10.1038 /$ ncomms 14221.

Lee, R. Y. N. et al. (1999) "EAT-4, a Homolog of a Mammalian Sodium-Dependent Inorganic Phosphate Cotransporter, Is Necessary for Glutamatergic Neurotransmission in Caenorhabditis elegans," The Journal of Neuroscience, 19(1), pp. 159-167. doi: 10.1523/JNEUROSCI.19-0100159.1999.

660 Li, J. et al. (2014) "Piezo1 integration of vascular architecture with physiological force," Nature, 661 515(7526). doi: 10.1038/nature13701.

$662 \mathrm{Li}, \mathrm{Z}$. et al. (2012) "Dissecting a central flip-flop circuit that integrates contradictory sensory cues in 663 C. elegans feeding regulation," Nature Communications, 3(1), p. 776. doi: 10.1038/ncomms1780. 664 Ma, S. et al. (2018) "Common PIEZO1 Allele in African Populations Causes RBC Dehydration and 665 Attenuates Plasmodium Infection,” Cell, 173(2). doi: 10.1016/j.cell.2018.02.047. 
666

667

668

669

670

671

672

673

674

675

676

677

678

679

680

681

682

683

684

685

686

687

688

689

690

691

692

693

694

695

696

697

698

699

700

701

702

703

704

705

706

707

708

709

710

711

712

713

714

715

716

717

Maksimovic, S. et al. (2014) "Epidermal Merkel cells are mechanosensory cells that tune mammalian touch receptors.," Nature, 509(7502), pp. 617-21. doi: 10.1038/nature13250. Mango, S. E. (2007) "The C. elegans pharynx: a model for organogenesis.," WormBook : the online review of C. elegans biology, pp. 1-26. doi: 10.1895/wormbook.1.129.1.

Martinac, B. et al. (1987) "Pressure-sensitive ion channel in Escherichia coli," Proceedings of the National Academy of Sciences of the United States of America, 84(8), pp. 2297-2301. doi: 10.1073/pnas.84.8.2297.

Min, S. et al. (2020) "Control of feeding by Piezo-mediated gut mechanosensation in Drosophila 1 2," bioRxiv, p. 2020.09.11.293712. Available at: https://doi.org/10.1101/2020.09.11.293712. Murthy, S. E. et al. (2018) "The mechanosensitive ion channel Piezo2 mediates sensitivity to mechanical pain in mice," Science Translational Medicine, 10(462), p. eaat9897. doi: 10.1126/scitranslmed.aat9897.

Niacaris, T. (2003) "Serotonin regulates repolarization of the C. elegans pharyngeal muscle," Journal of Experimental Biology, 206(2), pp. 223-231. doi: 10.1242/jeb.00101.

O'Hagan, R., Chalfie, M. and Goodman, M. B. (2005) "The MEC-4 DEG/ENaC channel of Caenorhabditis elegans touch receptor neurons transduces mechanical signals.," Nature neuroscience, 8(1), pp. 43-50. doi: 10.1038/nn1362.

Ohmachi, M. et al. (1999) "kel-1, a novel Kelch-related gene in Caenorhabditis elegans, is expressed in pharyngeal gland cells and is required for the feeding process," Genes to Cells, 4(6), pp. 325-337. doi: 10.1046/j.1365-2443.1999.00264.x.

Pan, B. et al. (2018) "TMC1 Forms the Pore of Mechanosensory Transduction Channels in Vertebrate Inner Ear Hair Cells.," Neuron, 99(4), pp. 736-753.e6. doi:

10.1016/j.neuron.2018.07.033.

Parpaite, T. and Coste, B. (2017) "Piezo channels," Current Biology, 27(7), pp. R250-R252. doi: 10.1016/j.cub.2017.01.048.

Pathak, M. M. et al. (2014) "Stretch-activated ion channel Piezo1 directs lineage choice in human neural stem cells.," Proceedings of the National Academy of Sciences of the United States of America, 111(45), pp. 16148-53. doi: 10.1073/pnas.1409802111.

Raizen, D. M. and Avery, L. (1994) "Electrical activity and behavior in the pharynx of caenorhabditis elegans," Neuron, 12(3), pp. 483-495. doi: 10.1016/0896-6273(94)90207-0. Raizen, D. M., Lee, R. Y. N. and Avery, L. (1995) "Interacting genes required for pharyngeal excitation by motor neuron MC in Caenorhabditis elegans," Genetics, 141(4), pp. 1365-1382. Ranade, S. S. et al. (2014) "Piezo2 is the major transducer of mechanical forces for touch sensation in mice.," Nature, 516(7529), pp. 121-5. doi: 10.1038/nature13980.

Retailleau, K. et al. (2015) "Piezo1 in Smooth Muscle Cells Is Involved in Hypertension-Dependent Arterial Remodeling.," Cell reports, 13(6), pp. 1161-1171. doi: 10.1016/j.celrep.2015.09.072.

Rode, B. et al. (2017) "Piezol channels sense whole body physical activity to reset cardiovascular homeostasis and enhance performance.," Nature communications, 8(1), p. 350. doi: 10.1038/s41467017-00429-3.

Romero, L. O. et al. (2019) "Dietary fatty acids fine-tune Piezol mechanical response," Nature Communications, 10(1), pp. 1-14. doi: 10.1038/s41467-019-09055-7.

Savage, C. et al. (1989) "mec-7 is a beta-tubulin gene required for the production of 15-

protofilament microtubules in Caenorhabditis elegans.," Genes \& development, 3(6), pp. 870-881. doi: 10.1101/gad.3.6.870.

Schindelin, J. et al. (2009) "Fiji - an Open platform for biological image analysis," Nature Methods, 9(7). doi: 10.1038/nmeth.2019.Fiji.

Schulenburg, H. and Félix, M. A. (2017) "The natural biotic environment of Caenorhabditis elegans," Genetics, 206(1), pp. 55-86. doi: 10.1534/genetics.116.195511.

Shtonda, B. B. and Avery, L. (2006) "Dietary choice behavior in Caenorhabditis elegans," Journal of Experimental Biology, 209(1), pp. 89-102. doi: 10.1242/jeb.01955.

Singh, R. N. and Sulston, J. E. (1978) "Some Observations On Moulting in Caenorhabditis Elegans," Nematologica, 24(1), pp. 63-71. doi: 10.1163/187529278X00074. 
718

719

720

721

722

723

724

725

726

727

728

729

730

731

732

733

734

735

736

737

738

739

740

741

742

743

744

745

746

747

748

749

750

751

752

753

754

755

756

757

758

759

760

761

762

Smit, R. B., Schnabel, R. and Gaudet, J. (2008) "The HLH-6 Transcription Factor Regulates C. elegans Pharyngeal Gland Development and Function," PLoS Genetics. Edited by S. E. Mango, 4(10), p. e1000222. doi: 10.1371/journal.pgen.1000222.

Strange, K., Christensen, M. and Morrison, R. (2007) "Primary culture of Caenorhabditis elegans developing embryo cells for electrophysiological, cell biological and molecular studies," Nature Protocols, 2(4), pp. 1003-1012. doi: 10.1038/nprot.2007.143.

Szczot, M. et al. (2018) "PIEZO2 mediates injury-induced tactile pain in mice and humans," Science Translational Medicine, 10(462), p. eaat9892. doi: 10.1126/scitranslmed.aat9892.

Taylor, S. R. et al. (2020) "Molecular topography of an entire nervous system," bioRxiv. doi: 10.1101/2020.12.15.422897.

Trojanowski, N. F., Raizen, D. M. and Fang-Yen, C. (2016) "Pharyngeal pumping in Caenorhabditis elegans depends on tonic and phasic signaling from the nervous system," Scientific Reports, 6(1), p. 22940. doi: 10.1038/srep22940.

Tsujimura, T. et al. (2019) "Involvement of the epithelial sodium channel in initiation of mechanically evoked swallows in anaesthetized rats," Journal of Physiology, 597(11). doi: 10.1113/JP277895.

Vidal-Gadea, A. G. et al. (2012) " Coordination of behavioral hierarchies during environmental transitions in Caenorhabditis elegans ," Worm, 1(1), pp. 5-11. doi: 10.4161/worm.19148.

Wang, P. et al. (2020) "Visceral Mechano-sensing Neurons Control Drosophila Feeding by Using Piezo as a Sensor," Neuron, pp. 1-11. doi: 10.1016/j.neuron.2020.08.017.

Wang, S. et al. (2016) "Endothelial cation channel PIEZO1 controls blood pressure by mediating flow-induced ATP release," Journal of Clinical Investigation, 126(12), pp. 4527-4536. doi: 10.1172/JCI87343.

Woo, S.-H. et al. (2014) "Piezo2 is required for Merkel-cell mechanotransduction.," Nature, 509(7502), pp. 622-6. doi: 10.1038/nature13251.

Woo, S.-H. et al. (2015) "Piezo2 is the principal mechanotransduction channel for proprioception," Nature Neuroscience, 18(12), pp. 1756-1762. doi: 10.1038/nn.4162.

Wu, J., Lewis, A. H. and Grandl, J. (2017) "Touch, Tension, and Transduction - The Function and Regulation of Piezo Ion Channels," Trends in Biochemical Sciences, 42(1), pp. 57-71. doi: 10.1016/j.tibs.2016.09.004.

Wu, J. et al. (2017) "Inactivation of Mechanically Activated Piezo1 Ion Channels Is Determined by the C-Terminal Extracellular Domain and the Inner Pore Helix". Cell Reports. 21(9) pp. 2357-2366. doi: 10.1016/j.celrep.2017.10.120.

Yamaguchi, T. et al. (2019) "PIEZO2 deficiency is a recognizable arthrogryposis syndrome: A new case and literature review," American Journal of Medical Genetics, Part A, 179(6). doi: 10.1002/ajmg.a.61142.

Yan, Z. et al. (2013) "Drosophila NOMPC is a mechanotransduction channel subunit for gentletouch sensation.," Nature, 493(7431), pp. 221-5. doi: 10.1038/nature11685.

$\mathrm{Yu}$, L. et al. (2015) "Bacterial respiration and growth rates affect the feeding preferences, brood size and lifespan of Caenorhabditis elegans," PLOS ONE, 10(7), pp. 1-13. doi:

10.1371/journal.pone.0134401.

Zarychanski, R. et al. (2012) "Mutations in the mechanotransduction protein PIEZO1 are associated with hereditary xerocytosis," Blood, 120(9), pp. 1908-1915. doi: 10.1182/blood-2012-04-422253. Zeng, W.-Z. et al. (2018) "PIEZOs mediate neuronal sensing of blood pressure and the baroreceptor reflex.,"Science (New York, N.Y.), 362(6413), pp. 464-467. doi: 10.1126/science.aau6324. 